\title{
Sur la normalité asymptotique d'un estimateur à noyau du quantile conditionnel pour des données censurées et associées
}

\section{On the asymptotic normality of a kernel conditional quantile estimator for censored and associated data}

\author{
Wafaa Djelladj ${ }^{1}$, Abdelkader Tatachak ${ }^{1}$ \\ 1'Laboratory MSTD, USTHB, Algiers, Algeria, wdjelladj@usthb.dz, atatachak@usthb.dz
}

RÉSUMÉ. L'objet du présent article est d'établir la normalité asymptotique d'un estimateur à noyau du quantile conditionnel dans un modèle de censure droite, pour lequel les durées de vie ainsi que les covariables sont supposées satisfaire une dépendance de type association.

ABSTRACT. This paper aims at establishing the asymptotic normality for a kernel conditional quantile estimator in a right censorship model for which, the lifetime observations and the covariates are assumed to satisfy an association dependency type.

MOTS-CLÉS. Association, données censurées, estimateur de Kaplan-Meier, normalité asymptotique, quantile conditionnel.

KEYWORDS. Association, asymptotic normality, censored data, conditional quantile, Kaplan-Meier estimator.

\section{Introduction}

In classical statistical inference, the observed random variables (rv's) of interest are generally assumed to be independent and identically distributed (iid). However, it is more common to have dependent variables in some real life situations. Dependent variables are present in several backgrounds such as medicine, biology and social sciences. Associated rv's are of considerable interest when dealing with survival analysis, reliability problems, percolation theory and some models in statistical mechanics.

In the literature, two kinds of dependency are widely used : mixing [DOUKHAN 1994] and association [ESARY et al. 1967]. These two concepts are not completely dissociated. In fact, we can find sequences that are associated but not mixing, associated and mixing, and mixing but not associated.

Recall that a set of finite family of rv's $\left(T_{1}, \ldots, T_{n}\right)$ which are defined on a common probability space $(\Omega, \mathscr{A}, \mathbb{P})$ are said to be associated if for every pair of non-decreasing componentwise functions $\Psi_{1}(\cdot)$ and $\Psi_{2}(\cdot)$ from $\mathbb{R}^{n}$ to $\mathbb{R}$,

$$
\operatorname{cov}\left(\Psi_{1}\left(T_{1}, \ldots, T_{n}\right), \Psi_{2}\left(T_{1}, \ldots, T_{n}\right)\right) \geq 0,
$$

whenever the covariance exists. An infinite family of rv's is associated if any finite sub-family is a set of associated rv's. The notion of association was firstly introduced by [ESARY et al. 1967] mainly for an application in reliability and its main advantage compared to mixing is that the conditions of limit theorems are easier to verify : indeed, a covariance is much easier to compute than a mixing coefficient. Let $\left\{T_{n}, n \geq 1\right\}$ be a strictly stationary sequence of associated rv's of interest having an unknown absolutely continuous distribution function (df) $F$. This variable can be considered as a lifetime under biomedical studies. The major characteristic of survival time is the incompleteness. Random right censoring is a well-known phenomenon in survival analysis since, the lifetime data may not be completely observable if the patient is still alive at the end of study, or is dead for another reason. Hence, the avai- 
lable data provide partial information. In this case, the variable of interest $T$ is subject to right censoring by another non-negative rv $C$. We assume that the censoring lifetimes are independent and identically distributed (iid) and possess an unknown $\mathrm{df} G$. We take in consideration the presence of a strictly stationary and associated covariate $\mathbf{X}$ taking values in $\mathbb{R}^{d}$. Under this model, the observable sequence is $\left\{\left(Y_{i}=\min \left(T_{i}, C_{i}\right), \delta_{i}=\mathbb{1}_{\left\{T_{i} \leq C_{i}\right\}}, \mathbf{X}_{i}\right) ; 1 \leq i \leq n\right\}$ where $\mathbb{1}_{A}$ denotes the indicator function of the event $A$. In order to ensure the identifiability of the model we assume that the censoring times $\left\{C_{i}, 1 \leq i \leq n\right\}$ are independent of $\left\{\left(\mathbf{X}_{i}, T_{i}\right), 1 \leq i \leq n\right\}$.

Under the model we consider here, we establish the asymptotic normality of the kernel conditional quantile estimator defined in (6). The rest of the paper is organized as follows : Section 1 gives the required notations as well as the estimators. The assumptions needed to state our results are gathered in Section 2 while Section 3 is devoted to the proofs of the main results.

\section{Estimators and notations}

The conditional df $F(t \mid x)$ can be written

$$
F(t \mid x)=\frac{F_{1}(x, t)}{l(x)}
$$

where

$$
F_{1}(x, t)=\frac{\partial F(x, .)}{\partial x}:=\frac{\partial^{d} F(x, .)}{\partial x_{1} \ldots \partial x_{d}}
$$

with $F(.,$.$) the joint df. The conditional quantile of T$ given $X=x$ for $p \in(0,1)$ is given by

$$
\xi_{p}(x)=\inf \{t, F(t \mid x) \geq p\} .
$$

In the complete data case (no censoring), the traditional kernel estimator of $F(t \mid \mathbf{x})$ is given by

$$
F_{1, n}(t \mid \mathbf{x})=\sum_{i=1}^{n} \omega_{i n}(\mathbf{x}) \mathbb{1}_{\left\{T_{i} \leq t\right\}},
$$

where $\omega_{i n}($.$) are measurable functions. These functions called weights were introduced by Nadaraya-$ Watson in the context of the kernel regression and defined by

$$
\omega_{i n}(\mathbf{x})=\frac{K_{d}\left(\frac{\mathbf{x}-\mathbf{X}_{i}}{h_{n, 1}}\right)}{\sum_{j=1}^{n} K_{d}\left(\frac{\mathbf{x}-\mathbf{X}_{j}}{h_{n, 1}}\right)}=\frac{\frac{1}{n h_{n, 1}^{d}} K_{d}\left(\frac{\mathbf{x}-\mathbf{X}_{i}}{h_{n, 1}}\right)}{l_{n}(\mathbf{x})},
$$

with the convention $0 / 0=0$. Here $K_{d}$ is a kernel function on $\mathbb{R}^{d}$ such that for any $\mathbf{x}_{l}=\left(x_{l}^{1}, x_{l}^{2}, \ldots, x_{l}^{d}\right)$, we have

$$
K_{d}\left(\frac{\mathbf{x}-\mathbf{x}_{l}}{h_{n, 1}}\right)=\prod_{i=1}^{d} K\left(\frac{x^{i}-x_{l}^{i}}{h_{n, 1}}\right),
$$


whereas $h_{n, 1}$ is a positive sequence of bandwidths tending to 0 along with $n$ and $l_{n}($.$) is the Parzen-$ Rosenblatt kernel estimator of $l($.$) .$

In the sequel, the weights are adapted to the censoring case, that is

$$
\omega_{i n}(\mathbf{x})=\frac{1}{n h_{n, 1}^{d}} \frac{\delta_{i}}{\bar{G}\left(Y_{i}\right) l_{n}(\mathbf{x})} K_{d}\left(\frac{\mathbf{x}-\mathbf{X}_{i}}{h_{n, 1}}\right) .
$$

The censoring df $G$ is usually unknown and its appropriate nonparametric estimator is the well-known Kaplan-Meier one, viz

$$
G_{n}(t)=1-\prod_{i=1}^{n}\left[1-\frac{1-\delta_{(i)}}{n-i+1}\right]^{\left.\mathbb{1}_{\left\{Y_{(i)} \leq t\right.}\right\}}
$$

where $Y_{(1)} \leq Y_{(2)} \leq \ldots \ldots \leq Y_{(n)}$ are the order statistics of $Y_{i}$ and $\delta_{(1)}, \delta_{(2)}, \ldots, \delta_{(n)}$ are the corresponding indicators of non censoring.

Using the weights defined in (4), [OULD SAID 2006] established a strong uniform consistency rate for the estimator in (3) in the iid case and $\mathrm{d}=1$. The smoothed version of $F_{\mathbb{1}, n}(\cdot \mid \cdot)$, known as « double kernel estimation approach » is

$$
F_{n}(t \mid \mathbf{x})=\frac{\frac{1}{n h_{n, 1}^{d}} \sum_{i=1}^{n} \frac{\delta_{i}}{\bar{G}_{n}\left(Y_{i}\right)} K_{d}\left(\frac{\mathbf{x}-\mathbf{X}_{i}}{h_{n, 1}}\right) H\left(\frac{t-Y_{i}}{h_{n, 2}}\right)}{\frac{1}{n h_{n, 1}^{d}} \sum_{i=1}^{n} K_{d}\left(\frac{\mathbf{x}-\mathbf{X}_{i}}{h_{n, 1}}\right)}=: \frac{F_{1, n}(\mathbf{x}, t)}{l_{n}(\mathbf{x})}
$$

The strong consistency and the asymptotic normality of this estimator was studied in the iid case and under $\alpha$-mixing condition by [OULD SAID and SADKI 2008, 2011]. Here, the bandwidth $h_{n, 2}$ is not necessarily equal to $h_{n, 1}$ and they will be denoted by $h_{1}:=h_{n, 1}$ and $h_{2}:=h_{n, 2}$.

Note that the estimator in (5) is an adapted version of that of [YU and JONES 1998] to the censoring case. Originally, this smooth estimate for complete data was proposed and discussed by the last authors mainly to avoid the crossing problem which occurs when using an indicator function instead of a continuous df. It follows that, in view of (5), a natural estimator of (2) can be computed by

$$
\xi_{p, n}(\mathbf{x})=\inf \left\{t, F_{n}(t \mid \mathbf{x}) \geq p\right\}
$$

Recall that censored-associated data was studied for the first time by [FERRANI et al. 2016] while the strong uniform consistency of the estimator under study was stated by [DJELLADJ and TATACHAK 2019]. Some supporting evidence show that in presence of outliers and for heavy-tailed or asymetric distributions, conditional quantiles can be helpful.

To overcome some difficulties encountered in computing some operators related to our estimators, the following pseudo estimate will be helpful in further calculations, namely

$$
\tilde{F}_{n}(t \mid x)=: \frac{\tilde{F}_{1, n}(x, t)}{l_{n}(x)}=\frac{\frac{1}{n h_{1}^{d}} \sum_{i=1}^{n} \frac{\delta_{i}}{\bar{G}\left(Y_{i}\right)} K_{d}\left(\frac{x-X_{i}}{h_{1}}\right) H\left(\frac{t-Y_{i}}{h_{2}}\right)}{\frac{1}{n h_{1}^{d}} \sum_{i=1}^{n} K_{d}\left(\frac{x-X_{i}}{h_{1}}\right)} .
$$


Thereafter, we will use the conditional pdf $f(t \mid$.$) defined by f(t \mid)=.\frac{\partial}{\partial t} F(t \mid$.$) . Hence, the corresponding$ estimators are given by

$$
f_{n}(t \mid x)=\frac{\frac{\partial}{\partial t} F_{1, n}(x, t)}{l_{n}(x)} \text { and } \quad \tilde{f}_{n}(t \mid x)=\frac{\frac{\partial}{\partial t} \tilde{F}_{1, n}(x, t)}{l_{n}(x)},
$$

where

$$
\frac{\partial}{\partial t} F_{1, n}(x, t)=: f_{n}(\mathbf{x}, t)=\frac{1}{n h_{1}^{d} h_{2}} \sum_{i=1}^{n} \frac{\delta_{i}}{\bar{G}_{n}\left(Y_{i}\right)} K_{d}\left(\frac{\mathbf{x}-\mathbf{X}_{i}}{h_{1}}\right) H^{(1)}\left(\frac{t-Y_{i}}{h_{2}}\right)
$$

and

$$
\frac{\partial}{\partial t} \tilde{F}_{1, n}(x, t)=: \tilde{f}_{n}(\mathbf{x}, t)=\frac{1}{n h_{1}^{d} h_{2}} \sum_{i=1}^{n} \frac{\delta_{i}}{\bar{G}\left(Y_{i}\right)} K_{d}\left(\frac{\mathbf{x}-\mathbf{X}_{i}}{h_{1}}\right) H^{(1)}\left(\frac{t-Y_{i}}{h_{2}}\right)
$$

where $H^{(1)}$ denotes the first derivative of $H$. Applying Taylor expansion of $F_{n}(. \mid$.$) in the neighborhood$ of $\xi_{p}$, we obtain

$$
F_{n}\left(\xi_{p, n}(x) \mid x\right)-F_{n}\left(\xi_{p}(x) \mid x\right)=\left(\xi_{p, n}(x)-\xi_{p}(x)\right) f_{n}\left(\xi_{p, n}^{*}(x) \mid x\right) .
$$

Here, $\xi_{p, n}^{*}$ lies between $\xi_{p}$ and $\xi_{p, n}$. This expansion permits to state asymptotic results for $\left(\xi_{p, n}(x)-\xi_{p}(x)\right)$ through those stated for $\left(F_{n}\left(\xi_{p, n}(x) \mid x\right)-F_{n}\left(\xi_{p}(x) \mid x\right)\right)$.

\section{Assumptions and main results}

In the sequel, $m_{1}$ stands for a positive constant taking different values and $\tau$ will denote a positive real number satisfying $\tau<\tau_{F}<\tau_{G}$ where, for any df $W, \tau_{W}:=\sup \{y ; W(y)<1\}$. Define $\Omega_{0}=\{\mathbf{x} \in$ $\left.\mathbb{R}^{d} / l(\mathbf{x}) \geq m_{0}:=\inf _{x} l(\mathbf{x})>0\right\}$ and let $\Omega$ and $\mathcal{C}$ be compact sets included in $\Omega_{0}$ and $[0, \tau]$, respectively. The main results will be stated using the following assumptions

A1. The bandwidths $h_{1}$ and $h_{2}$ satisfy
(i) $h_{1} \rightarrow 0, n h_{1}^{2 \alpha+d(1-\alpha)} \rightarrow+\infty$ with $\alpha \in(0,1)$ and $\frac{\log ^{5} n}{n h_{1}^{d}} \rightarrow 0$ as $n \rightarrow+\infty$,
(ii) $h_{2} \rightarrow 0, n h_{1}^{d} h_{2} \rightarrow+\infty$ as $n \rightarrow+\infty$,
(iii) $n h_{1}^{d} h_{2}^{4} \rightarrow 0$ and $n h_{1}^{d+4} \rightarrow 0$ as $n \rightarrow+\infty$,
(iv) $v_{s} h_{1}^{d} \rightarrow 0$ with $v_{s}$ a sequence of real numbers;

A2. The kernel $K_{d}$ is a bounded pdf, compactly supported and satisfies :

(i) $K_{d}$ is Hölder continuous of order $\alpha$,

(ii) $\int_{\mathbb{R}^{d}} u_{j} K_{d}(\mathbf{u}) \mathbf{d u}=0$, for all $j=1, \ldots, d$, where $\mathbf{u}=\left(u_{1}, \ldots, u_{d}\right)^{\top}$,

(iii) The kernel $K_{d}$ has bounded partial first derivatives;

A3. The function $H$ in (5) is of class $\mathcal{C}^{1}$. Furthermore, its derivative $H^{(1)}$ is assumed to be compactly supported and satisfies the properties of a second order kernel and

(i) $\int_{\mathbb{R}}|t|^{\alpha} H^{(1)}(t) d t<+\infty$;

A4. The marginal density $l($.$) is bounded and twice differentiable with \sup _{\mathbf{x} \in \Omega}\left|\frac{\partial^{k} l(\mathbf{x})}{\partial x_{i} \partial x_{j}^{k-1}}(x)\right|<\infty$ for $i, j=1, \ldots, d$ and $k=1,2$; 
A5. The joint pdf $f(.,$.$) is bounded and differentiable up to order 3, moreover$

(i)The conditional df $F(t \mid x)$ satrisfies the lipschitz condition of order $u_{1}$ and $u_{2}$ with respect to $\mathbf{x}$ and $t$ and

$\forall\left(\mathbf{x}_{1}, \mathbf{x}_{2}\right) \in \Omega^{2}, \forall\left(t_{1}, t_{2}\right) \in \mathbb{R}^{2},\left|F\left(t_{1} \mid \mathbf{x}_{1}\right)-F\left(t_{2} \mid \mathbf{x}_{2}\right)\right| \leq m_{1}\left(\left|\mathbf{x}_{1}-\mathbf{x}_{2}\right|^{u_{1}}+\left|t_{1}-t_{2}\right|^{u_{2}}\right)$,

(ii) $\forall \mathbf{x} \in \Omega$, we have that $\int_{\mathbb{R}}|t| f(t \mid \mathbf{x}) d t<+\infty$;

A6. The joint pdf $l_{i, j}(.,$.$) of \left(\mathbf{X}_{i}, \mathbf{X}_{j}\right)$ is bounded;

A7. The joint pdf $f(., ., .,$.$) of \left(\mathbf{X}_{i}, Y_{i}, \mathbf{X}_{j}, Y_{j}\right)$ is bounded;

A8. Let us define $\Lambda_{i j}$ as follows

$$
\Lambda_{i j}:=\sum_{k=1}^{d} \sum_{l=1}^{d} \operatorname{Cov}\left(X_{i}^{k}, X_{j}^{l}\right)+2 \sum_{k=1}^{d} \operatorname{Cov}\left(X_{i}^{k}, Y_{j}\right)+\operatorname{Cov}\left(Y_{i}, Y_{j}\right),
$$

with $X_{i}^{k}$ the k-th component of $\mathbf{X}_{i}$, such that for all $j \geq 1$ and $r>0$

$$
\sup _{i:|j-i| \geq r} \Lambda_{i j}=: \rho(r) \leq \gamma_{0} e^{-\gamma r}, \text { for all } \gamma_{0}, \gamma>0
$$

A9. The function $\varsigma(\mathbf{x})=\int_{\mathbb{R}} \frac{1}{\bar{G}(v)} f(\mathbf{x}, v) d v$ is bounded, continuously differentiable and $\sup _{\mathbf{x} \in \Omega}\left|\frac{\partial \varsigma}{\partial x_{i}}(\mathbf{x})\right|<$ $\infty$ for $i=1, \ldots, d$.

We need the following assumptions for the asymptotic normality

N1. The survival df $G$ of censored rv's has a bounded first derivative $g$;

N2. Let $0<p_{n}<n, 0<q_{n}<n$ be integers tending to $\infty$ with $\mathrm{n}$ such that $p_{n}+q_{n} \leq n$. Let $k_{n}$ be the largest integer for which $k_{n}\left(p_{n}+q_{n}\right) \leq n$ and

(i) $\frac{k_{n} p_{n}}{n} \rightarrow 1$ (ii) $p_{n} h_{1}^{d} \rightarrow 0$ and $\frac{p_{n}^{2}}{n h_{1}^{d}} \rightarrow 0$ (iii) $\frac{e^{-\gamma q_{n}}}{h_{1}^{d+2} h_{2}^{2}} \rightarrow 0$ as $n \rightarrow \infty$.

REMARK 1- Assumption A1 gives a classical choice of the bandwidths in functional estimation. For the sake of simplicity, many authors consider that $h_{1}=h_{2}$ which is not justified in general. Note that the condition $\boldsymbol{A 1}$ ( ii) implies the first condition in $\boldsymbol{A 1}(i)$ if $d \geq 2$. For $d=1$, the comparison is not straightforward and depends upon the order of magnitude of $h_{2}$ with respect to $h_{1}^{\alpha}$. Assumption A2 is quite usual in kernel estimation. Assumptions A3-A7 are classical in nonparametric estimation under dependency and $\mathbf{A 5}(i)$ and (ii) are used in the calculation of the variance term in asymptotic normality while $\mathbf{A} 8$ is used for covariance calculation under association structure. Furthermore, this assumption gives a progressive trend to asymptotic independence of "past" and "future". Note that Assumption A9 is mainly technical. Assumption N1 is used in technical calculations of the asymptotic normality. Finally, Assumption N2 is especially usefull in the case of dependent data when studying the asymptotic normality and is used when dealing with the technique of big and small blocks, $\mathbf{N} 2(i)$ and the fact that $\frac{k_{n}\left(p_{n}+q_{n}\right)}{n} \rightarrow 1$ imply that $\frac{k_{n} q_{n}}{n} \rightarrow 0$. Remark that $\frac{k_{n} q_{n} / n}{k_{n} p_{n} / n} \rightarrow 0$ gives that $q_{n}<p_{n}$. Add to this the first part of Assumption $\mathbf{N 2}\left(\right.$ ii), we get that $q_{n} h_{n}^{d} \rightarrow 0$.

THEOREM 1- Under assumptions A1-A8 and N1-N2, for any $x \in \Omega_{0}$ and $l(\boldsymbol{x})>0$, we have

$$
\sqrt{n h_{1}^{d}}\left(F_{n}(t \mid \boldsymbol{x})-F(t \mid \boldsymbol{x})\right) \stackrel{\mathcal{D}}{\longrightarrow} \mathcal{N}\left(0, \sigma^{2}(\boldsymbol{x}, t)\right) \quad \text { as } n \rightarrow+\infty
$$

with $D$ the convergence in distribution. The variance is such that

$$
\sigma^{2}(\boldsymbol{x}, t)=\frac{\kappa F(t \mid \boldsymbol{x})(1-\bar{G}(t) F(t \mid \boldsymbol{x}))}{l(\boldsymbol{x}) \bar{G}(t)}
$$


and $\kappa=\int_{\mathbb{R}^{d}} K_{d}^{2}(\boldsymbol{u}) d \boldsymbol{u}<+\infty$.

COROLlARY $1-$ Assume that $p \in(0,1)$. Under assumptions of Theorem 1 and for any $\boldsymbol{x} \in \Omega_{0}$ such that $f\left(\xi_{p}(\boldsymbol{x}) \mid \boldsymbol{x}\right) \neq 0$, we have

$$
\sqrt{n h_{1}^{d}}\left(\xi_{p, n}(\boldsymbol{x})-\xi_{p}(\boldsymbol{x})\right) \stackrel{\mathcal{D}}{\longrightarrow} \mathcal{N}\left(0, \sigma_{\xi}^{2}\left(\boldsymbol{x}, \xi_{p}(\boldsymbol{x})\right)\right) \quad \text { as } n \rightarrow+\infty
$$

with

$$
\sigma_{\xi}^{2}\left(\boldsymbol{x}, \xi_{p}(\boldsymbol{x})\right)=\frac{\sigma^{2}\left(\boldsymbol{x}, \xi_{p}(\boldsymbol{x})\right)}{f^{2}\left(\xi_{p}(\boldsymbol{x}) \mid \boldsymbol{x}\right)}
$$

REMARK 2- If we replace $l(),. f(. \mid),. \bar{G}($.$) and \xi_{p}($.$) by their estimators l_{n}(),. f_{n}(. \mid),. \bar{G}_{n}($.$) and \xi_{p, n}($.$) ,$ respectively then a plug-in type convergent estimator denoted $\sigma_{\xi, n}^{2}\left(\boldsymbol{x}, \xi_{p, n}(\boldsymbol{x})\right)$ of $\sigma_{\xi}^{2}\left(\boldsymbol{x}, \xi_{p}(\boldsymbol{x})\right)$ is easily obtained. Note that

$$
\sigma_{\xi, n}^{2}\left(\boldsymbol{x}, \xi_{p, n}(\boldsymbol{x})\right)=\frac{\kappa F_{n}(t \mid \boldsymbol{x})\left(1-\bar{G}_{n}(t) F_{n}(t \mid \boldsymbol{x})\right)}{l_{n}(\boldsymbol{x}) \bar{G}_{n}(t) f_{n}^{2}\left(\xi_{p, n}(\boldsymbol{x}) \mid \boldsymbol{x}\right)}
$$

Using Corollary 1, the approximate $(1-\vartheta)$ confidence interval is then given by

$$
\left[\xi_{p, n}-z_{1-\vartheta / 2} \frac{\sigma_{\xi, n}\left(\boldsymbol{x}, \xi_{p, n}(\boldsymbol{x})\right)}{\sqrt{n h_{1}^{d}}}, \xi_{p, n}+z_{1-\vartheta / 2} \frac{\sigma_{\xi, n}\left(\boldsymbol{x}, \xi_{p, n}(\boldsymbol{x})\right)}{\sqrt{n h_{1}^{d}}}\right]
$$

with $z_{1-\vartheta / 2}$ stands for the $(1-\vartheta / 2)$-quantile of the standard normal distribution.

\section{Proofs of the main results}

We first deal with the uniform a.s. convergence of the conditional pdf estimator $f_{n}(t \mid \mathbf{x})$ to $f(t \mid \mathbf{x})$ defined in (7).

Proposition 1- Under assumptions A1-A8, we have

$$
\sup _{\boldsymbol{x} \in \Omega} \sup _{t \in \mathcal{C}}\left|f_{n}(t \mid \boldsymbol{x})-f(t \mid \boldsymbol{x})\right| \rightarrow 0 \text { a.s. as } n \rightarrow+\infty \text {. }
$$

\subsection{Proof of Proposition 1}

To prove the convergence of the underlying pdf, we use the following decomposition

$$
f_{n}(t \mid \mathbf{x})-f(t \mid \mathbf{x})=\left(f_{n}(t \mid \mathbf{x})-\tilde{f}_{n}(t \mid \mathbf{x})\right)+\left(\tilde{f}_{n}(t \mid \mathbf{x})-f(t \mid \mathbf{x})\right)
$$

We firstly deal with the left hand side of (10). Using Assumption A3, we find

$$
\left|f_{n}(t \mid \mathbf{x})-\tilde{f}_{n}(t \mid \mathbf{x})\right|=\frac{1}{l_{n}(\mathbf{x})}\left|f_{n}(\mathbf{x}, t)-\tilde{f}_{n}(\mathbf{x}, t)\right|
$$




$$
\leq \frac{m_{1}}{l_{n}(\mathbf{x})} \frac{\sup _{t \in \mathcal{C}}\left|\bar{G}_{n}(t)-\bar{G}(t)\right|}{\bar{G}_{n}\left(\tau_{F}\right) \bar{G}\left(\tau_{F}\right)} \frac{1}{n h_{1}^{d} h_{2}} \sum_{i=1}^{n} K_{d}\left(\frac{\mathbf{x}-\mathbf{X}_{i}}{h_{1}}\right) H^{(1)}\left(\frac{t-Y_{i}}{h_{2}}\right) .
$$

Then, we get immediately the convergence of the left hand side of (10). As for the right hand side of (10), we have

$$
\begin{aligned}
\sup _{\mathbf{x} \in \Omega} \sup _{t \in \mathcal{C}}\left|\tilde{f}_{n}(t \mid \mathbf{x})-f(t \mid \mathbf{x})\right| \leq & \frac{1}{\inf _{\mathbf{x} \in \Omega}\left(l_{n}(\mathbf{x})\right)}\left\{\sup _{\mathbf{x} \in \Omega} \sup _{t \in \mathcal{C}}\left|\tilde{f}_{n}(\mathbf{x}, t)-\mathbb{E}\left[\tilde{f}_{n}(\mathbf{x}, t)\right]\right|\right. \\
& +\sup _{\mathbf{x} \in \Omega} \sup _{t \in \mathcal{C}}\left|\mathbb{E}\left[\tilde{f}_{n}(\mathbf{x}, t)\right]-f_{n}(\mathbf{x}, t)\right| \\
& \left.+\sup _{\mathbf{x} \in \Omega}\left|l_{n}(\mathbf{x})-l(\mathbf{x})\right| \sup _{\mathbf{x} \in \Omega} \sup _{t \in \mathcal{C}}|f(t \mid \mathbf{x})|\right\} \\
= & : \frac{1}{m_{0}-\sup _{\mathbf{x} \in \Omega}\left|l_{n}(\mathbf{x})-l(\mathbf{x})\right|}\left[I_{1}+I_{2}+I_{3}\right] .
\end{aligned}
$$

Let us deal with each term of the right hand side. As for $I_{1}$, we make choice of the following expression

$$
\begin{aligned}
Q_{i}(\mathbf{x}, t) & =\frac{1}{n h_{1}^{d} h_{2}} \frac{\delta_{i}}{\bar{G}\left(Y_{i}\right)} K_{d}\left(\frac{\mathbf{x}-\mathbf{X}_{i}}{h_{1}}\right) H^{(1)}\left(\frac{t-Y_{i}}{h_{2}}\right) \\
& -\mathbb{E}\left[\frac{1}{n h_{1}^{d} h_{2}} \frac{\delta_{i}}{\bar{G}\left(Y_{i}\right)} K_{d}\left(\frac{\mathbf{x}-\mathbf{X}_{i}}{h_{1}}\right) H^{(1)}\left(\frac{t-Y_{i}}{h_{2}}\right)\right]
\end{aligned}
$$

such that

$$
\sum_{i=1}^{n} Q_{i}(\mathbf{x}, t)=\tilde{f}_{n}(\mathbf{x}, t)-\mathbb{E}\left[\tilde{f}_{n}(\mathbf{x}, t)\right] .
$$

The proof uses the covering techniques. The compact subset $\mathcal{C}$ is covered by a finite number $\mu_{n}$ of intervals of length $\varrho_{n}$, respectively centered at $t_{1}, \ldots, t_{\mu_{n}}$ with $\mu_{n} \varrho_{n} \leq c$. There exists $t_{j}$ for any t such that $\left|t-t_{j}\right| \leq \varrho_{n}$.

$$
\left|\sum_{i=1}^{n} Q_{i}(\mathbf{x}, t)\right| \leq\left|\sum_{i=1}^{n} Q_{i}(\mathbf{x}, t)-\sum_{i=1}^{n} Q_{i}\left(\mathbf{x}, t_{j}\right)\right|+\left|\sum_{i=1}^{n} Q_{i}\left(\mathbf{x}, t_{j}\right)\right|
$$

It follows from assumptions $\mathbf{A 2}$ and $\mathbf{A} \mathbf{3}$ that

$$
\begin{aligned}
\left|\sum_{i=1}^{n} Q_{i}(\mathbf{x}, t)-\sum_{i=1}^{n} Q_{i}\left(\mathbf{x}, t_{j}\right)\right| \leq & \frac{2}{n h_{1}^{d} h_{2}} \sum_{i=1}^{n} \frac{\delta_{i}}{\bar{G}\left(Y_{i}\right)} K_{d}\left(\frac{\mathbf{x}-\mathbf{X}_{i}}{h_{1}}\right) \\
& \times\left|H^{(1)}\left(\frac{t-Y_{i}}{h_{2}}\right)-H^{(1)}\left(\frac{t_{j}-Y_{i}}{h_{2}}\right)\right| \\
\leq & \frac{m_{1}}{h_{1}^{d} h_{2} \bar{G}\left(\tau_{F}\right)}\left|\frac{t-t_{j}}{h_{2}}\right| \\
\leq & \frac{m_{1}}{h_{1}^{d} h_{2}^{2} \mu_{n}}
\end{aligned}
$$


By means of (11) and choosing $\mu_{n}=O(n)$, we get

$$
I_{1} \leq \frac{m_{1}}{n h_{1}^{d} h_{2}^{2}}+\max _{1 \leq j \leq \mu_{n}}\left|\sum_{i=1}^{n} Q_{i}\left(\mathbf{x}, t_{j}\right)\right|
$$

It is now to be shown that

$$
\mathbb{P}\left(\max _{1 \leq j \leq \mu_{n}}\left|\sum_{i=1}^{n} Q_{i}\left(\mathbf{x}, t_{j}\right)\right|>\varepsilon\right) \rightarrow 0 \text { as } n \rightarrow+\infty
$$

As $Q_{i}\left(\mathbf{x}, t_{j}\right)$ are associated, the use of an exponential inequality due to [DOUKHAN and NEUMANN 2007] is appropriate to bound (12). An analogous framework as in the proof of the consistency above is established. We proceed now to the demonstration of the bias term $I_{2}$.

It is easily seen that

$$
\mathbb{E}\left[\tilde{f}_{n}(\mathbf{x}, t)\right]=\frac{1}{h_{1}^{d} h_{2}} \mathbb{E}\left[K_{d}\left(\frac{\mathbf{x}-\mathbf{X}_{1}}{h_{1}}\right) \mathbb{E}\left[\frac{\delta_{1}}{\bar{G}\left(Y_{1}\right)} H^{(1)}\left(\frac{t-Y_{1}}{h_{2}}\right) \mid \mathbf{X}_{1}\right]\right]
$$

We have

$$
\begin{aligned}
\mathbb{E}\left[\frac{\delta_{1}}{\bar{G}\left(Y_{1}\right)} H^{(1)}\left(\frac{t-Y_{1}}{h_{2}}\right) \mid \mathbf{X}_{1}\right] & =\mathbb{E}\left[\mathbb{E}\left[\frac{\delta_{1}}{\bar{G}\left(Y_{1}\right)} H^{(1)}\left(\frac{t-Y_{1}}{h_{2}}\right) \mid T_{1}\right] \mid \mathbf{X}_{1}\right] \\
& =h_{2} \int_{\mathbb{R}} H^{(1)}(z) f\left(t-h_{2} z \mid \mathbf{X}_{1}\right) d z .
\end{aligned}
$$

Therefore

$$
\begin{aligned}
\mathbb{E}\left[\tilde{f}_{n}(\mathbf{x}, t)\right] & =\frac{1}{h_{1}^{d} h_{2}} \int_{\mathbb{R}^{d}} \int_{\mathbb{R}} h_{2} K_{d}\left(\frac{\mathbf{x}-u}{h_{1}}\right) H^{(1)}(z) f\left(t-h_{2} z \mid \mathbf{u}\right) l(\mathbf{u}) d \mathbf{u} d z \\
& =\int_{\mathbb{R}^{d}} \int_{\mathbb{R}} K_{d}(\mathbf{r}) H^{(1)}(z) f\left(\mathbf{x}-h_{1} \mathbf{r}, t-h_{2} z\right) d r d z .
\end{aligned}
$$

with $f\left(\mathbf{x}-h_{1} \mathbf{r}, t-h_{2} z\right)=F_{1}^{\prime}\left(\mathbf{x}-h_{1} \mathbf{r}, t-h_{2} z\right)$. Assumption $\mathbf{A 5}$ and a Taylor expansion of $f(.,$.$) around$ $(\mathbf{x}, t)$ gives

$$
\begin{aligned}
f\left(\mathbf{x}-r h_{1}, t-z h_{2}\right) & =f(\mathbf{x}, t)-h_{1}\left[r_{1} \frac{\partial f(\mathbf{x}, t)}{\partial x_{1}}+\cdots+r_{d} \frac{\partial f(\mathbf{x}, t)}{\partial x_{d}}\right]-h_{2}\left[z \frac{\partial f(\mathbf{x}, t)}{\partial t}\right] \\
& +\frac{h_{1}^{2}}{2}\left[r_{1}^{2} \frac{\partial^{2} f\left(\mathbf{x}^{*}, t\right)}{\partial x_{1}^{2}}+\cdots+r_{d}^{2} \frac{\partial^{2} f\left(\mathbf{x}^{*}, t\right)}{\partial x_{d}^{2}}+2 \sum_{i \neq j} r_{i} r_{j} \frac{\partial^{2} f\left(\mathbf{x}^{*}, t\right)}{\partial x_{i} \partial x_{j}}\right] \\
& +\frac{h_{2}^{2}}{2}\left[z^{2} \frac{\partial^{2} f\left(\mathbf{x}, t^{*}\right)}{\partial t^{2}}\right]+h_{1} h_{2}\left[r_{1} z \frac{\partial^{2} f\left(\mathbf{x}^{*}, t\right)}{\partial x_{1} \partial t}+\cdots+r_{d} z \frac{\partial^{2} f\left(\mathbf{x}^{*}, t\right)}{\partial x_{d} \partial t}\right] .
\end{aligned}
$$


Note that $\mathbf{x}^{*}$ lies between $\mathbf{x}-r h_{1}$ and $\mathbf{x}$ and $t^{*}$ between $t-z h_{2}$ and $t$. By assumptions $\mathbf{A 2}$ and A5 $I_{2}=O\left(h_{1}^{2}+h_{2}^{2}\right)$. As for $I_{3}$, under assumptions A1, A2, A4, A6 and A8, it follows from Lemma 3 in [MENNI and TATACHAK 2018] that the kernel estimator $l_{n}(\mathbf{x}) \rightarrow l(\mathbf{x})$ a.s. as $n \rightarrow+\infty$. Add to this the first part of Assumption A5 then we get $I_{3}=o(1)$. This point ends the proof of Proposition 1 .

\subsection{Proof of Theorem 1}

We have the following decomposition

$$
F_{n}(t \mid \mathbf{x})-F(t \mid \mathbf{x})=\left(F_{n}(t \mid \mathbf{x})-\tilde{F}_{n}(t \mid \mathbf{x})\right)+\left(\tilde{F}_{n}(t \mid \mathbf{x})-F(t \mid \mathbf{x})\right) .
$$

And

$$
\begin{aligned}
\tilde{F}_{n}(t \mid \mathbf{x})-F(t \mid \mathbf{x}) & =\frac{l(\mathbf{x})}{l_{n}(\mathbf{x})}\left[\frac{\tilde{F}_{1, n}(\mathbf{x}, t)-\mathbb{E}\left(\tilde{F}_{1, n}(\mathbf{x}, t)\right)-F(t \mid \mathbf{x})\left(l_{n}(\mathbf{x})-\mathbb{E}\left(l_{n}(\mathbf{x})\right)\right)}{l(\mathbf{x})}\right] \\
& -\left[\frac{F_{1}(\mathbf{x}, t)-\mathbb{E}\left(\tilde{F}_{1, n}(\mathbf{x}, t)\right)-F(t \mid \mathbf{x})\left(l(\mathbf{x})-\mathbb{E}\left(l_{n}(\mathbf{x})\right)\right)}{l_{n}(\mathbf{x})}\right] \\
& =: \frac{l(\mathbf{x})}{l_{n}(\mathbf{x})} Z_{n}(\mathbf{x}, t)-R_{n}(\mathbf{x}, t) .
\end{aligned}
$$

Note that

$$
\sqrt{n h_{1}^{d}}\left(F_{n}(t \mid \mathbf{x})-F(t \mid \mathbf{x})\right)=: \frac{l(\mathbf{x})}{l_{n}(\mathbf{x})}\left[\sqrt{n h_{1}^{d}} Z_{n}(\mathbf{x}, t)\right]+\sqrt{n h_{1}^{d}} U_{n}(\mathbf{x}, t)
$$

with

$$
U_{n}(\mathbf{x}, t)=:\left(F_{n}(t \mid \mathbf{x})-\tilde{F}_{n}(t \mid \mathbf{x})\right)-R_{n}(\mathbf{x}, t)
$$

The idea is to give asymptotic results of $\sqrt{n h_{1}^{d}} Z_{n}(\mathbf{x}, t)$. For this puspose, we give the convergence in probability of the negligible term $\sqrt{n h_{1}^{d}} U_{n}(\mathbf{x}, t)$ to zero as shown in Lemma 1 and determine the asymptotic variance that appears in (8) (see Lemma 3). Finally, we use the Bernstein's procedure of big blocks and small blocks to obtain the asymptotic normality of the principal term $Z_{n}(\mathbf{x}, t)$. Regarding the term $\frac{l(\mathbf{x})}{l_{n}(\mathbf{x})}$, it converges obviously to 1 .

The next lemma deals with the second part of (14).

LEMMA 1- Assumptions A1-A5 and A8 yield that

$$
\sqrt{n h_{1}^{d}} U_{n}(\boldsymbol{x}, t)=o_{\mathbb{P}}(1) \text { as } n \rightarrow+\infty
$$

\subsection{Proof of Lemma 1}

Observe that (15) can be written

$$
\sqrt{n h_{1}^{d}} U_{n}(\mathbf{x}, t)=\frac{\sqrt{n h_{1}^{d}}}{l_{n}(\mathbf{x})}\left[\left(F_{1, n}(\mathbf{x}, t)-\tilde{F}_{1, n}(\mathbf{x}, t)\right)-\left(F_{1}(\mathbf{x}, t)-\mathbb{E}\left(\tilde{F}_{1, n}(\mathbf{x}, t)\right)\right]\right.
$$




$$
\begin{array}{ll}
+ & \frac{\sqrt{n h_{1}^{d}}}{l_{n}(\mathbf{x})}\left[F(t \mid \mathbf{x})\left(l(\mathbf{x})-\mathbb{E}\left(l_{n}(\mathbf{x})\right)\right)\right] \\
=: & \mathcal{X}_{n, 1}+\mathcal{X}_{n, 2}+\mathcal{X}_{n, 3} .
\end{array}
$$

The convergence rate of each term in (16) follows thanks to lemmas 5.6, 5.5 and 5.4 in [DJELLADJ and TATACHAK 2019], respectively. In fact, using A1(iii),(iv) we show that

$\mathcal{X}_{n, 1}=o_{\mathbb{P}}\left(\sqrt{n^{1-2 \theta} h_{1}^{d}}\right)=: o_{\mathbb{P}}\left(\sqrt{v_{s} h_{1}^{d}}\right)=o_{\mathbb{P}}(1)$

$\mathcal{X}_{n, 2}=O\left(\sqrt{n h_{1}^{d}}\left(h_{1}^{2}+h_{2}^{2}\right)\right)=o(1)$

$\mathcal{X}_{n, 3}=O\left(\sqrt{n h_{1}^{d+4}}\right)=o(1)$.

This achieves the proof of Lemma 1.

Let us deal with the asymptotic normality of the main term $Z_{n}(\mathbf{x}, t)$. For this we first set

$$
\Psi_{i}(\mathbf{x}, t)=K_{d, i}\left[H_{i} \frac{\delta_{i}}{\bar{G}\left(Y_{i}\right)}-F(t \mid \mathbf{x})\right]-\mathbb{E}\left[K_{d, i}\left(H_{i} \frac{\delta_{i}}{\bar{G}\left(Y_{i}\right)}-F(t \mid \mathbf{x})\right)\right]
$$

with

$$
K_{d, i}:=K_{d}\left(\frac{\mathbf{x}-\mathbf{X}_{i}}{h_{1}}\right) \text { and } H_{i}:=H\left(\frac{t-Y_{i}}{h_{2}}\right)
$$

Then we write

$$
Z_{n}(\mathbf{x}, t)=: \frac{1}{n h_{1}^{d} l(\mathbf{x})} \sum_{i=1}^{n} \Psi_{i}(\mathbf{x}, t)
$$

Now, our interest concerns the variance term

$$
\begin{aligned}
n h_{1}^{d} \operatorname{Var} Z_{n}(\mathbf{x}, t) & =\frac{1}{h_{1}^{d} l^{2}(\mathbf{x})} \operatorname{Var}\left(\Psi_{1}(\mathbf{x}, t)\right)+\frac{1}{n h_{1}^{d} l^{2}(\mathbf{x})} \sum_{i=1}^{n} \sum_{j=1: j \neq i}^{n} \operatorname{cov}\left(\Psi_{i}(\mathbf{x}, t), \Psi_{j}(\mathbf{x}, t)\right) \\
& =\sigma_{n}^{2}(\mathbf{x}, t)+\Xi_{n}(\mathbf{x}, t) .
\end{aligned}
$$

The next lemma reports intermediary results used in further calculations related to the variance term.

LEMMA 2- Assumptions A3(i), A5(i),(ii) and N1 give that

$$
\operatorname{Var}\left[\frac{\delta_{1}}{\bar{G}\left(Y_{1}\right)} H\left(\frac{t-Y_{1}}{h_{2}}\right) \mid \boldsymbol{X}_{1}\right] \rightarrow F(t \mid \boldsymbol{x})\left[\frac{1}{\bar{G}(t)}-F(t \mid \boldsymbol{x})\right] \text { as } n \rightarrow+\infty
$$

\subsection{Proof of Lemma 2}

We have

$$
\operatorname{Var}\left[\frac{\delta_{1}}{\bar{G}\left(Y_{1}\right)} H\left(\frac{t-Y_{1}}{h_{2}}\right) \mid \mathbf{X}_{1}\right]=\mathbb{E}\left[\frac{\delta_{1}}{\bar{G}^{2}\left(Y_{1}\right)} H^{2}\left(\frac{t-Y_{1}}{h_{2}}\right) \mid \mathbf{X}_{1}\right]-\mathbb{E}\left[\frac{\delta_{1}}{\bar{G}\left(Y_{1}\right)} H\left(\frac{t-Y_{1}}{h_{2}}\right) \mid \mathbf{X}_{1}\right]^{2}
$$




$$
=: \mathcal{L} 1+\mathcal{L} 2
$$

Integration by parts and change of variable give

$$
\begin{aligned}
\mathbb{E}\left[\frac{\delta_{1}}{\bar{G}\left(Y_{1}\right)} H\left(\frac{t-Y_{1}}{h_{2}}\right) \mid \mathbf{X}_{1}\right] & =\mathbb{E}\left[\frac{1}{\bar{G}\left(T_{1}\right)} H\left(\frac{t-T_{1}}{h_{2}}\right) \mathbb{E}\left[\mathbb{1}_{\left\{T_{1} \leq C_{1}\right\}} \mid T_{1}\right] \mid \mathbf{X}_{1}\right] \\
& =\int_{\mathbb{R}} H\left(\frac{t-u}{h_{2}}\right) f\left(u \mid \mathbf{X}_{1}\right) d u \\
& =\int_{\mathbb{R}} H^{(1)}(z) F\left(t-z h_{2} \mid \mathbf{X}_{1}\right) d z \\
& =\int_{\mathbb{R}} H^{(1)}(z)\left(F\left(t-z h_{2} \mid \mathbf{X}_{1}\right)-F(t \mid \mathbf{x})\right) d z+\int_{\mathbb{R}} H^{(1)}(z) F(t \mid \mathbf{x}) d z
\end{aligned}
$$

Clearly, we have $\int_{\mathbb{R}} H^{(1)}(z) F(t \mid \mathbf{x}) d z \rightarrow F(t \mid \mathbf{x})$. Regarding the left hand side, by assumptions A3(i) and A5(i), we have that

$$
\begin{aligned}
\int_{\mathbb{R}} H^{(1)}(z)\left(F\left(t-z h_{2} \mid \mathbf{X}_{1}\right)-F(t \mid \mathbf{x})\right) d z \leq & \int_{\mathbb{R}} H^{(1)}(z) m_{1}\left(\left|\mathbf{X}_{1}-\mathbf{x}\right|^{u_{1}}+\left|z h_{2}\right|^{u_{2}}\right) d z \\
= & m_{1}\left|\mathbf{X}_{1}-\mathbf{x}\right|^{u_{1}} \int_{\mathbb{R}} H^{(1)}(z) d z \\
& +m_{1}\left|h_{2}\right|^{u_{2}} \int_{\mathbb{R}}|z|^{u_{2}} H^{(1)}(z) d z \\
= & O\left(h_{2}^{\min \left(u_{1}, u_{2}\right)}\right) .
\end{aligned}
$$

Hence $\mathcal{L}_{2} \rightarrow F^{2}(t \mid \mathbf{x})$ as $n \rightarrow+\infty$. Regarding $\mathcal{L}_{1}$, by a change of variable

$$
\begin{aligned}
\mathcal{L}_{1} & =\mathbb{E}\left[\frac{1}{\bar{G}^{2}\left(T_{1}\right)} H^{2}\left(\frac{t-T_{1}}{h_{2}}\right) \mathbb{E}\left[\mathbb{1}_{\left\{T_{1} \leq C_{1}\right\}} \mid T_{1}\right] \mid \mathbf{X}_{1}\right] \\
& =\int_{\mathbb{R}} \frac{1}{\bar{G}(y)} H^{2}\left(\frac{t-y}{h_{2}}\right) f\left(y \mid \mathbf{X}_{1}\right) d y \\
& =\int_{\mathbb{R}} h_{2} \frac{1}{\bar{G}\left(t-z h_{2}\right)} H^{2}(z) f\left(t-z h_{2} \mid \mathbf{X}_{1}\right) d z .
\end{aligned}
$$

Now, we use a Taylor expansion around $t$. We get

$$
\begin{aligned}
\mathcal{L}_{1} & =\frac{h_{2}}{\bar{G}(t)} \int_{\mathbb{R}} H^{2}(z) f\left(t-z h_{2} \mid \mathbf{X}_{1}\right) d z-\frac{h_{2}^{2}}{\bar{G}^{2}(t)} g\left(t^{*}\right) \int_{\mathbb{R}} z H^{2}(z) f\left(t-z h_{2} \mid \mathbf{X}_{1}\right) d z \\
& =: \mathcal{A}_{1}-\mathcal{A}_{2} .
\end{aligned}
$$


Bounding $\mathcal{A}_{2}$ gives

$$
\mathcal{A}_{2} \leq \frac{h_{2}^{2} \sup _{t \in \mathcal{C}} g(t)}{\bar{G}^{2}\left(\tau_{F}\right)} \int_{\mathbb{R}}|z| f\left(t-z h_{2} \mid \mathbf{X}_{1}\right) d z
$$

Applying assumptions A5(ii) and N1, we conclude that $\mathcal{A}_{2}=O\left(h_{2}^{2}\right)$. By an integration by parts on the term $\mathcal{A}_{1}$, we get

$$
\begin{aligned}
\mathcal{A}_{1} & =\frac{1}{\bar{G}(t)} \int_{\mathbb{R}} 2 H^{(1)}(z) H(z) F\left(t-z h_{2} \mid \mathbf{X}_{1}\right) d z \\
& =\frac{1}{\bar{G}(t)} \int_{\mathbb{R}} 2 H^{(1)}(z) H(z)\left(F\left(t-z h_{2} \mid \mathbf{X}_{1}\right)-F(t \mid \mathbf{x})\right) d z+\frac{1}{\bar{G}(t)} \int_{\mathbb{R}} 2 H^{(1)}(z) H(z) F(t \mid \mathbf{x}) d z
\end{aligned}
$$

As well as above, we use Assumption A5(i). Then

$$
\begin{aligned}
\int_{\mathbb{R}} 2 H^{(1)}(z) H(z)\left(F\left(t-z h_{2} \mid \mathbf{X}_{1}\right)-F(t \mid \mathbf{x})\right) d z & \leq \int_{\mathbb{R}} m_{1}\left(\left|\mathbf{X}_{1}-\mathbf{x}\right|^{u_{1}}+\left|z h_{2}\right|^{u_{2}}\right)\left[H(z)^{2}\right]^{\prime} d z \\
& =O\left(h_{2}^{\min \left(u_{1}, u_{2}\right)}\right) .
\end{aligned}
$$

Moreover

$$
\int_{\mathbb{R}} 2 H^{(1)}(z) H(z) F(t \mid \mathbf{x}) d z=F(t \mid \mathbf{x}) .
$$

Hence $\mathcal{A}_{1} \rightarrow \frac{F(t \mid \mathbf{x})}{\bar{G}(t)}$ as $n \rightarrow \infty$. We deduce that

$$
\operatorname{Var}\left[\frac{\delta_{1}}{\bar{G}\left(Y_{1}\right)} H\left(\frac{t-Y_{1}}{h_{2}}\right) \mid \mathbf{X}_{1}\right] \rightarrow \frac{F(t \mid \mathbf{x})}{\bar{G}(t)}-O\left(h_{2}^{2}\right)-F(t \mid \mathbf{x})^{2}
$$

which concludes the proof of Lemma 2.

LEMMA 3- Under assumptions A2-A4 and A6-A7, we have

$$
\sigma_{n}^{2}(\boldsymbol{x}, t) \rightarrow \sigma^{2}(\boldsymbol{x}, t) \text { as } n \rightarrow+\infty \quad \text { and } \quad \Xi_{n}(\boldsymbol{x}, t) \rightarrow 0 \text { as } n \rightarrow+\infty
$$

\subsection{Proof of Lemma 3}

From (18), we define

$$
\sigma_{n}^{2}(\mathbf{x}, t)=\frac{1}{h_{1}^{d} l^{2}(\mathbf{x})} \mathbb{E}\left[\Psi_{1}^{2}(\mathbf{x}, t)\right]
$$




$$
\begin{aligned}
= & \frac{1}{h_{1}^{d} l^{2}(\mathbf{x})} \mathbb{E}\left[K_{d, 1}^{2}\left(H_{1} \frac{\delta_{1}}{\bar{G}\left(Y_{1}\right)}-F(t \mid \mathbf{x})\right)^{2}\right] \\
& -\frac{1}{h_{1}^{d} l^{2}(\mathbf{x})}\left\{\mathbb{E}\left[K_{d, 1}\left(H_{1} \frac{\delta_{1}}{\bar{G}\left(Y_{1}\right)}-F(t \mid \mathbf{x})\right)\right]\right\}^{2} \\
= & \mathcal{R}_{1}(\mathbf{x}, t)-\mathcal{R}_{2}(\mathbf{x}, t) .
\end{aligned}
$$

As for $\mathcal{R}_{2}(\mathbf{x}, t)$, we can write

$$
\begin{aligned}
\mathcal{R}_{2}(\mathbf{x}, t) & =\frac{1}{h_{1}^{d} l^{2}(\mathbf{x})}\left[\mathbb{E}\left(\frac{\delta_{1}}{\bar{G}\left(Y_{1}\right)} K_{d, 1} H_{1}\right)-\mathbb{E}\left(K_{d, 1} F(t \mid \mathbf{x})\right)\right]^{2} \\
& =\frac{1}{h_{1}^{d} l^{2}(\mathbf{x})}\left[h_{1}^{d} \mathbb{E}\left(\tilde{F}_{1, n}(\mathbf{x}, t)\right)-h_{1}^{d} F(t \mid \mathbf{x}) \mathbb{E}\left(l_{n}(\mathbf{x})\right)\right]^{2} \\
& =\frac{1}{l^{2}(\mathbf{x})}\left[\mathbb{E}\left(\tilde{F}_{1, n}(\mathbf{x}, t)\right)-F(t \mid \mathbf{x}) \mathbb{E}\left(l_{n}(\mathbf{x})\right)\right]^{2}
\end{aligned}
$$

We point out that

$$
\mathbb{E}\left(\tilde{F}_{1, n}(\mathbf{x}, t)\right)-F(t \mid \mathbf{x}) \mathbb{E}\left(l_{n}(\mathbf{x})\right) \rightarrow F_{1}(\mathbf{x}, t)-F(t \mid \mathbf{x}) \mathbb{E}\left(l_{n}(\mathbf{x})\right) \text { as } n \rightarrow \infty .
$$

Then

$$
F_{1}(\mathbf{x}, t)-F(t \mid \mathbf{x}) \mathbb{E}\left(l_{n}(\mathbf{x})\right)=F(t \mid \mathbf{x})\left[l(\mathbf{x})-\mathbb{E}\left(l_{n}(\mathbf{x})\right)\right] \rightarrow 0 \text { as } n \rightarrow \infty .
$$

On the other hand

$$
\begin{aligned}
\mathcal{R}_{1}(\mathbf{x}, t)= & \frac{1}{h_{1}^{d} l^{2}(\mathbf{x})} \mathbb{E}\left[\mathbb{E}\left[K_{d, 1}^{2}\left(H_{1} \frac{\delta_{1}}{\bar{G}\left(Y_{1}\right)}-F(t \mid \mathbf{x})\right)^{2} \mid \mathbf{X}_{1}\right]\right] \\
= & \frac{1}{h_{1}^{d} l^{2}(\mathbf{x})} \mathbb{E}\left[K_{d, 1}^{2} \operatorname{Var}\left(H_{1} \frac{\delta_{1}}{\bar{G}\left(Y_{1}\right)} \mid \mathbf{X}_{1}\right)\right] \\
& +\frac{1}{h_{1}^{d} l^{2}(\mathbf{x})} \mathbb{E}\left[K_{d, 1}^{2} \mathbb{E}\left(\left(H_{1} \frac{\delta_{1}}{\bar{G}\left(Y_{1}\right)} \mid \mathbf{X}_{1}\right)-F(t \mid \mathbf{x})\right)^{2}\right] .
\end{aligned}
$$

Working as in lemma 2, the second part in brackets tends to zero as $n$ tends to infinity. In terms of the first part, we write

$$
\begin{aligned}
\mathbb{E}\left[K_{d, 1}^{2} \operatorname{Var}\left(H_{1} \frac{\delta_{1}}{\bar{G}\left(Y_{1}\right)} \mid \mathbf{X}_{1}\right)\right] & =\mathbb{E}\left[K_{d, 1}^{2} F(t \mid \mathbf{x})\left(\frac{1}{\bar{G}(t)}-F(t \mid \mathbf{x})\right)\right] \\
& =F(t \mid \mathbf{x})\left(\frac{1-\bar{G}(t) F(t \mid \mathbf{x})}{\bar{G}(t)}\right) \int_{\mathbb{R}^{d}} K_{d}^{2}\left(\frac{\mathbf{x}-\mathbf{u}}{h_{1}}\right) l(\mathbf{u}) d \mathbf{u} .
\end{aligned}
$$

Using a change of variable, Assumption $\mathbf{A 3}$ and Taylor expansion around $\mathbf{x}$, we get

$$
\int_{\mathbb{R}^{d}} K_{d}^{2}\left(\frac{\mathbf{x}-\mathbf{u}}{h_{1}}\right) l(\mathbf{u}) d \mathbf{u}=h_{1}^{d} \int_{\mathbb{R}^{d}} K_{d}^{2}(\mathbf{z}) l\left(\mathbf{x}-h_{1} \mathbf{z}\right) d \mathbf{z}
$$


Then

$$
\begin{aligned}
\frac{1}{h_{1}^{d} l^{2}(\mathbf{x})} \mathbb{E}\left[K_{d, 1}^{2} \operatorname{Var}\left(H_{1} \frac{\delta_{1}}{\bar{G}\left(Y_{1}\right)} \mid \mathbf{X}_{1}\right)\right] \rightarrow & \frac{1}{l(\mathbf{x})} F(t \mid \mathbf{x})\left(\frac{1-\bar{G}(t) F(t \mid \mathbf{x})}{\bar{G}(t)}\right) \int_{\mathbb{R}^{d}} K_{d}^{2}(\mathbf{z}) d \mathbf{z} \\
& =\frac{\kappa F(t \mid \mathbf{x})(1-\bar{G}(t) F(t \mid \mathbf{x}))}{l(\mathbf{x}) \bar{G}(t)}=\sigma^{2}(\mathbf{x}, t) .
\end{aligned}
$$

Now we deal with the second part of (18). We have

$$
\left.\Xi_{n}(\mathbf{x}, t)=\frac{1}{n h_{1}^{d} l^{2}(\mathbf{x})} \sum_{i=1}^{n} \sum_{j=1: j \neq i}^{n} \operatorname{cov}\left(\Psi_{i}(\mathbf{x}, t), \Psi_{j}(\mathbf{x}, t)\right)\right),
$$

which we rewrite

$$
\begin{aligned}
\Xi_{n}(\mathbf{x}, t) & \left.=\frac{1}{n h_{1}^{d} l^{2}(\mathbf{x})} \sum_{i=1}^{n} \sum_{B_{1}} \operatorname{cov}\left(\Psi_{i}(\mathbf{x}, t), \Psi_{j}(\mathbf{x}, t)\right)\right)+\frac{1}{n h_{1}^{d} l^{2}(\mathbf{x})} \sum_{i=1}^{n} \sum_{B_{2}} \operatorname{cov}\left(\Psi_{i}(\mathbf{x}, t), \Psi_{j}(\mathbf{x}, t)\right) \\
& =: \mathcal{M}_{1}+\mathcal{M}_{2}
\end{aligned}
$$

with

$$
B_{1}=\left\{(i, l) ; 1 \leq|i-l| \leq \eta_{n}\right\} \text { and } B_{2}=\left\{(i, l) ; \eta_{n}+1 \leq|i-l| \leq n-1\right\}
$$

Regarding $\mathcal{M}_{1}$, considering that the covariance is computed according to formula

$$
\begin{aligned}
\left.\operatorname{cov}\left(\Psi_{i}(\mathbf{x}, t), \Psi_{j}(\mathbf{x}, t)\right)\right)= & \left.\mathbb{E}\left[\Psi_{i}(\mathbf{x}, t) . \Psi_{j}(\mathbf{x}, t)\right)\right] \\
\leq & \mathbb{E}\left[K_{d, i} K_{d, j}\left(H_{i} \frac{\delta_{i}}{\bar{G}\left(Y_{i}\right)}-F(t \mid \mathbf{x})\right)\left(H_{j} \frac{\delta_{j}}{\bar{G}\left(Y_{j}\right)}-F(t \mid \mathbf{x})\right)\right] \\
& +\mathbb{E}\left[K_{d, 1}\left(H_{1} \frac{\delta_{1}}{\bar{G}\left(Y_{1}\right)}-F(t \mid \mathbf{x})\right)\right]^{2} .
\end{aligned}
$$

Clearly, we have

$$
\left|H_{i} \frac{\delta_{i}}{\bar{G}\left(Y_{i}\right)}-F(t \mid \mathbf{x})\right| \leq \frac{1}{\bar{G}\left(\tau_{F}\right)}+1
$$

Consequently

$$
\left.\operatorname{cov}\left(\Psi_{i}(\mathbf{x}, t), \Psi_{j}(\mathbf{x}, t)\right)\right) \leq\left(\frac{1}{\bar{G}\left(\tau_{F}\right)}+1\right)^{2} \mathbb{E}\left(K_{d, i} K_{d, j}\right)+O(1) \mathbb{E}\left(K_{d, 1}\right)^{2}
$$

Assumptions A2 and A6 give

$$
\begin{aligned}
\mathbb{E}\left(K_{d, i} K_{d, j}\right) & =\int_{\mathbb{R}^{d}} \int_{\mathbb{R}^{d}} K_{d}\left(\frac{\mathbf{x}-\mathbf{u}}{h_{1}}\right) K_{d}\left(\frac{\mathbf{x}-\mathbf{v}}{h_{1}}\right) l_{i, j}(\mathbf{u}, \mathbf{v}) d \mathbf{u} d \mathbf{v} \\
& =h_{1}^{2 d} \int_{\mathbb{R}^{d}} \int_{\mathbb{R}^{d}} K_{d}(\mathbf{s}) K_{d}(\mathbf{z}) l_{i, j}\left(\mathbf{x}-h_{1} \mathbf{s}, \mathbf{x}-h_{1} \mathbf{z}\right) d \mathbf{s} d \mathbf{z}
\end{aligned}
$$




$$
=O\left(h_{1}^{2 d}\right)
$$

Again, by a Taylor expansion and assumptions A2(i) and A4, we have that $\mathbb{E}\left(K_{d, 1}\right)=O\left(h_{1}^{d}\right)$. Bounding $\mathcal{M}_{1}$ gives

$$
\begin{aligned}
\mathcal{M}_{1} & \leq \frac{1}{n h_{1}^{d} l^{2}(\mathbf{x})} \sum_{i=1}^{n} \sum_{B_{1}}\left[m_{1}\left(\frac{1}{\bar{G}\left(\tau_{F}\right)}+1\right)^{2} h_{1}^{2 d}+O(1) h_{1}^{2 d}\right] \\
& =m_{1} \frac{h_{1}^{d} \eta_{n}}{l^{2}(\mathbf{x})}+O(1) \frac{h_{1}^{d} \eta_{n}}{l^{2}(\mathbf{x})}
\end{aligned}
$$

Supposing $h_{1}^{d} \eta_{n} \rightarrow 0$ as $n \rightarrow+\infty$, we get $\mathcal{M}_{1}=o(1)$.

The second term can be written

$$
\begin{aligned}
\mathcal{M}_{2} & =\frac{1}{n h_{1}^{d} l^{2}(\mathbf{x})} \sum_{i=1}^{n} \sum_{B_{2}} \operatorname{cov}\left(K_{d, i} H_{i} \frac{\delta_{i}}{\bar{G}\left(Y_{i}\right)}-K_{d, i} F(t \mid \mathbf{x}), K_{d, j} H_{j} \frac{\delta_{j}}{\bar{G}\left(Y_{j}\right)}-K_{d, j} F(t \mid \mathbf{x})\right) \\
& =: \frac{1}{n h_{1}^{d} l^{2}(\mathbf{x})} \sum_{i=1}^{n} \sum_{B_{2}} \mathcal{W} .
\end{aligned}
$$

We have

$$
\begin{aligned}
\mathcal{W} & =\operatorname{cov}\left(K_{d, i} H_{i} \frac{\delta_{i}}{\bar{G}\left(Y_{i}\right)}, K_{d, j} H_{j} \frac{\delta_{j}}{\bar{G}\left(Y_{j}\right)}\right)-F(t \mid \mathbf{x}) \operatorname{cov}\left(K_{d, i} H_{i} \frac{\delta_{i}}{\bar{G}\left(Y_{i}\right)}, K_{d, j}\right) \\
& -F(t \mid \mathbf{x}) \operatorname{cov}\left(K_{d, i}, K_{d, j} H_{j} \frac{\delta_{j}}{\bar{G}\left(Y_{j}\right)}\right)+F(t \mid \mathbf{x})^{2} \operatorname{cov}\left(K_{d, i}, K_{d, j}\right) \\
& =: \mathcal{W}_{1}-F(t \mid \mathbf{x}) \mathcal{W}_{2}-F(t \mid \mathbf{x}) \mathcal{W}_{3}+F(t \mid \mathbf{x})^{2} \mathcal{W}_{4} .
\end{aligned}
$$

Note that

$$
\begin{aligned}
\frac{1}{n h_{1}^{d} l^{2}(\mathbf{x})} \sum_{i=1}^{n} \sum_{B_{2}} \mathcal{W}_{1} & \leq \frac{1}{n h_{1}^{d} l^{2}(\mathbf{x})} \sum_{i=1}^{n} \sum_{B_{2}} m_{1} h_{1}^{d} h_{2}^{\frac{2}{d+1}} \rho^{\frac{d}{2 d+2}}(|i-j|) \\
& \leq \frac{m_{1}}{l^{2}(\mathbf{x})} \sum_{B_{2}} h_{2}^{\frac{2}{d+1}} \gamma_{0}^{\frac{d}{2 d+2}} e^{-\frac{\gamma|i-j| d}{2 d+2}} \\
& \leq \frac{m_{1} h_{2}^{\frac{2}{d+1}}}{l^{2}(\mathbf{x})} \int_{\eta_{n}}^{n} e^{-\frac{\gamma u d}{2 d+2}} d u \\
& \leq O\left(h_{2}^{\frac{2}{d+1}} e^{-\frac{\gamma \eta_{n} d}{2 d+2}}\right) .
\end{aligned}
$$

Choosing $\eta_{n}=O\left(h_{2}^{\nu-1}\right), 0<\nu<1$, (19) is of order $o\left(h_{2}^{\frac{2}{d+1}}\right)$.

As for $\mathcal{W}_{2}$ and $\mathcal{W}_{3}$, we have

$$
\mathcal{W}_{2}=\mathbb{E}\left[K_{d, i} K_{d, j} H_{i} \frac{\delta_{i}}{\bar{G}\left(Y_{i}\right)}\right]-\mathbb{E}\left[K_{d, i} H_{i} \frac{\delta_{i}}{\bar{G}\left(Y_{i}\right)}\right] \mathbb{E}\left[K_{d, j}\right]
$$




$$
=: \mathcal{W}_{2}^{\prime}-\mathcal{W}_{2}^{\prime \prime}
$$

Using conditional expectation techniques, we get

$$
\begin{aligned}
\mathcal{W}_{2}^{\prime} & =\mathbb{E}\left[K_{d}\left(\frac{\mathbf{x}-\mathbf{X}_{i}}{h_{1}}\right) K_{d}\left(\frac{\mathbf{x}-\mathbf{X}_{j}}{h_{1}}\right) \mathbb{E}\left[H\left(\frac{t-Y_{i}}{h_{2}}\right) \frac{\delta_{i}}{\bar{G}\left(Y_{i}\right)} \mid \mathbf{X}_{i}, \mathbf{X}_{j}\right]\right] \\
& =\mathbb{E}\left[K_{d}\left(\frac{\mathbf{x}-\mathbf{X}_{i}}{h_{1}}\right) K_{d}\left(\frac{\mathbf{x}-\mathbf{X}_{j}}{h_{1}}\right) \mathbb{E}\left[\mathbb{E}\left(\frac{\mathbb{1}_{\left\{T_{i} \leq C_{i}\right\}}}{\bar{G}\left(Y_{i}\right)} H\left(\frac{t-Y_{i}}{h_{2}}\right) \mid T_{i}\right) \mid \mathbf{X}_{i}, \mathbf{X}_{j}\right]\right] \\
& =\int_{\mathbb{R}^{d}} \int_{\mathbb{R}^{d}} \int_{\mathbb{R}} K_{d}\left(\frac{\mathbf{x}-\mathbf{u}}{h_{1}}\right) K_{d}\left(\frac{\mathbf{x}-\mathbf{v}}{h_{1}}\right) H\left(\frac{t-s}{h_{2}}\right) f(\mathbf{u}, \mathbf{v}, s) d \mathbf{u} d \mathbf{v} d s
\end{aligned}
$$

and

$$
\begin{aligned}
\mathcal{W}_{2}^{\prime \prime} & =\mathbb{E}\left[K_{d}\left(\frac{\mathbf{x}-\mathbf{X}_{i}}{h_{1}}\right) \mathbb{E}\left(\frac{\delta_{i}}{\bar{G}\left(Y_{i}\right)} H\left(\frac{t-Y_{i}}{h_{2}}\right) \mid \mathbf{X}_{i}\right)\right] \mathbb{E}\left[K_{d}\left(\frac{\mathbf{x}-\mathbf{X}_{j}}{h_{1}}\right)\right] \\
& =\mathbb{E}\left[K_{d}\left(\frac{\mathbf{x}-\mathbf{X}_{i}}{h_{1}}\right) \mathbb{E}\left(\mathbb{E}\left(\frac{\mathbb{1}_{\left\{T_{i} \leq C_{i}\right\}}}{\bar{G}\left(Y_{i}\right)} H\left(\frac{t-Y_{i}}{h_{2}}\right) \mid T_{i}\right) \mid \mathbf{X}_{i}\right)\right] \mathbb{E}\left[K_{d}\left(\frac{\mathbf{x}-\mathbf{X}_{j}}{h_{1}}\right)\right] \\
& =\int_{\mathbb{R}^{d}} \int_{\mathbb{R}} K_{d}\left(\frac{\mathbf{x}-\mathbf{u}}{h_{1}}\right) H\left(\frac{t-s}{h_{2}}\right) f(\mathbf{u}, s) d \mathbf{u} d s \int_{\mathbb{R}^{d}} K_{d}\left(\frac{\mathbf{x}-\mathbf{v}}{h_{1}}\right) l(\mathbf{v}) d \mathbf{v} .
\end{aligned}
$$

Using assumptions (A2), (A7), a change of variables and the fact that $H$ is bounded by 1 , subtracting gives

$$
\begin{aligned}
\mathcal{W}_{2}^{\prime}-\mathcal{W}_{2}^{\prime \prime} & =\iint_{\mathbb{R}^{d}} \int_{\mathbb{R}^{d}} \int_{\mathbb{R}} K_{d}\left(\frac{\mathbf{x}-\mathbf{u}}{h_{1}}\right) K_{d}\left(\frac{\mathbf{x}-\mathbf{v}}{h_{1}}\right) H\left(\frac{t-s}{h_{2}}\right)[f(\mathbf{u}, \mathbf{v}, s)-f(\mathbf{u}, s) l(\mathbf{v})] d \mathbf{u} d \mathbf{v} d s \\
& =O\left(h_{1}^{2 d} h_{2}\right) .
\end{aligned}
$$

In the same way, by assumptions (A2), (A4) and $\mathbf{A 6}$ we get

$$
\begin{aligned}
\mathcal{W}_{4} & =\mathbb{E}\left[K_{d, i} K_{d, j}\right]-\mathbb{E}\left[K_{d, i}\right] \mathbb{E}\left[K_{d, j}\right] \\
& =\iint_{\mathbb{R}^{d}} \int_{\mathbb{R}^{d}} K_{d}\left(\frac{\mathbf{x}-\mathbf{u}}{h_{1}}\right) K_{d}\left(\frac{\mathbf{x}-\mathbf{v}}{h_{1}}\right)[l(\mathbf{u}, \mathbf{v})-l(\mathbf{u}) l(\mathbf{v})] d \mathbf{u} d \mathbf{v} \\
& =O\left(h_{1}^{2 d}\right) .
\end{aligned}
$$

All these steps conclude that

$$
\Xi_{n}(\mathbf{x}, t) \rightarrow 0 \text { as } n \rightarrow+\infty
$$

which achives the proof of Lemma 3.

By the means of (14) and Lemma 3, to justify the Theorem 1, it suffices to show that

$$
\sqrt{n h_{1}^{d}}\left(Z_{n}(\mathbf{x}, t)\right) \stackrel{\mathcal{D}}{\longrightarrow} \mathcal{N}\left(0, \sigma^{2}(\mathbf{x}, t)\right) \text { as } n \rightarrow+\infty .
$$


To do that, as already mentioned above, we use the Bernstein's procedure based on small and big blocks. In the sequel, for the sake of simplify, we will normalize $\psi_{i}$. Therefore, from (17), we get

$$
\begin{aligned}
\sqrt{n h_{1}^{d}}\left(Z_{n}(\mathbf{x}, t)\right) & =\frac{1}{\sqrt{n}} \sum_{i=1}^{n} \frac{\Psi_{i}(\mathbf{x}, t)}{\sqrt{h_{1}^{d} l(\mathbf{x})}} \\
& =: \frac{1}{\sqrt{n}} \sum_{i=1}^{n} \tilde{\Psi}_{i}(\mathbf{x}, t) \\
& =: \frac{\tilde{S}_{n}}{\sqrt{n}} .
\end{aligned}
$$

Moreover, it can be checked from (18) and Lemma 3 that

$$
\operatorname{var}\left(\tilde{\psi}_{i}\right)=\frac{\operatorname{var}\left(\psi_{i}\right)}{h_{1}^{d} l^{2}(\mathbf{x})}=: \sigma_{n}^{2}(\mathbf{x}, t) \text { and } \operatorname{var}\left(\tilde{\psi}_{i}\right) \rightarrow \sigma^{2}(\mathbf{x}, t) \text { as } n \rightarrow+\infty .
$$

Note also that

$$
\sum_{i=1}^{n} \sum_{j=1: j \neq i}^{n} \operatorname{cov}\left(\tilde{\Psi}_{i}(\mathbf{x}, t), \tilde{\Psi}_{j}(\mathbf{x}, t)\right)=\frac{1}{h_{1}^{d} l^{2}(\mathbf{x})} \sum_{i=1}^{n} \sum_{j=1: j \neq i}^{n} \operatorname{cov}\left(\Psi_{i}(\mathbf{x}, t), \Psi_{j}(\mathbf{x}, t)\right)=o(1) .
$$

Clearly, (20) is equivalent to

$$
\frac{\tilde{S_{n}}}{\sqrt{n}} \stackrel{\mathcal{D}}{\longrightarrow} \mathcal{N}\left(0, \sigma^{2}(\mathbf{x}, t)\right) \text { as } n \rightarrow+\infty
$$

The main goal is to establish the asymptotic normality of $\frac{\tilde{S_{n}}}{\sqrt{n}}$. To this end we use the Bernstein's blocking technique. Let us partition the set $\{1, \ldots, n\}$ into $2 k_{n}+1$ subsets and for $m=1, \ldots, k_{n}$, we set

$$
\begin{aligned}
& I_{m}=\left\{i ; i=(m-1)\left(p_{n}+q_{n}\right)+1, \ldots,(m-1)\left(p_{n}+q_{n}\right)+p_{n}\right\} \\
& J_{m}=\left\{j ; j=(m-1)\left(p_{n}+q_{n}\right)+p_{n}+1, \ldots, m\left(p_{n}+q_{n}\right)\right\} .
\end{aligned}
$$

The remaining points are defined in the set $\left\{l ; k_{n}\left(p_{n}+q_{n}\right)+1 \leq l \leq n\right\}$ which may be empty and $p_{n}$, $q_{n}$ and $k_{n}$ are given in Assumption N2. Let us define the rv's $U_{n m}, U_{n m}^{\prime}$ and $U_{n m}^{\prime \prime}$ as follows

$$
U_{n m}=\sum_{i=(m-1)\left(p_{n}+q_{n}\right)+1}^{(m-1)\left(p_{n}+q_{n}\right)+p_{n}} \tilde{\Psi}_{i}, \quad U_{n m}^{\prime}=\sum_{j=(m-1)\left(p_{n}+q_{n}\right)+p_{n}+1}^{m\left(p_{n}+q_{n}\right)} \tilde{\Psi}_{j}, U_{n k}^{\prime \prime}=\sum_{l=k_{n}\left(p_{n}+q_{n}\right)+1}^{n} \tilde{\Psi}_{l}
$$

with

$$
\begin{aligned}
\frac{\tilde{S}_{n}}{\sqrt{n}} & =\frac{1}{\sqrt{n}}\left[\sum_{m=1}^{k_{n}} U_{n m}+\sum_{m=1}^{k_{n}} U_{n m}^{\prime}+U_{n k_{n}}^{\prime \prime}\right] \\
& =: \frac{1}{\sqrt{n}}\left[T_{n}+T_{n}{ }^{\prime}+T_{n}{ }^{\prime \prime}\right] .
\end{aligned}
$$


Then, in order to establish the convergence of (21), we have to show that

$$
\frac{T_{n}}{\sqrt{n}} \stackrel{\mathcal{D}}{\longrightarrow} \mathcal{N}\left(0, \sigma^{2}(\mathbf{x}, t)\right) \quad \text { as } n \rightarrow+\infty
$$

and

$$
\frac{1}{n} \mathbb{E}\left[T_{n}^{\prime 2}\right]+\frac{1}{n} \mathbb{E}\left[T_{n}^{\prime \prime 2}\right] \rightarrow 0 \text { as } n \rightarrow+\infty
$$

In order to prove (22) and (23), we use the following lemmas

LEMMA 4- Assumption N2 yields that

$$
\begin{aligned}
& \text { (i) } \frac{k_{n}}{n} \operatorname{var}\left(U_{n 1}^{\prime}\right) \rightarrow 0 \\
& \text { (ii) } \frac{1}{n}\left|\operatorname{cov}\left(U_{n 1}^{\prime}, U_{n, l+1}^{\prime}\right)\right| \leq \frac{q_{n} m_{1}}{n l^{2}(\boldsymbol{x})} \frac{1}{h_{1}^{d+2} h_{2}^{2}} \sum_{r=l\left(p_{n}+q_{n}\right)-\left(q_{n}-1\right)}^{l\left(p_{n}+q_{n}\right)+\left(q_{n}-1\right)}\left|\Lambda_{1, r+1}(\boldsymbol{x}, t)\right| \\
& \text { (iii) } \frac{1}{n} \sum_{1 \leq i<j \leq k_{n}}|\operatorname{cov}|\left(U_{n i}^{\prime}, U_{n j}^{\prime}\right) \mid \rightarrow 0
\end{aligned}
$$

\subsection{Proof of Lemma 4}

(i) We have

$$
\begin{aligned}
\frac{k_{n}}{n} \operatorname{var}\left(U_{n 1}^{\prime}\right) & =\frac{k_{n}}{n} \operatorname{var}\left(\sum_{j=p_{n}+1}^{p_{n}+q_{n}} \tilde{\Psi}_{j}(\mathbf{x}, t)\right) \\
& =\frac{k_{n} q_{n}}{n} \operatorname{var}\left(\tilde{\Psi}_{1}(\mathbf{x}, t)\right)+\frac{2 k_{n}}{n} \sum_{1 \leq i<j \leq q_{n}}\left|\operatorname{cov}\left(\tilde{\Psi}_{i}(\mathbf{x}, t), \tilde{\Psi}_{j}(\mathbf{x}, t)\right)\right| \\
& =\frac{k_{n} q_{n}}{n} \frac{1}{h_{1}^{d} l^{2}(\mathbf{x})} \operatorname{var}\left(\Psi_{1}(\mathbf{x}, t)\right)+\frac{2 k_{n}}{n} \sum_{1 \leq i<j \leq q_{n}}\left|\operatorname{cov}\left(\tilde{\Psi}_{i}(\mathbf{x}, t), \tilde{\Psi}_{j}(\mathbf{x}, t)\right)\right| \\
& =: \mathcal{J}_{1}+\mathcal{J}_{2} .
\end{aligned}
$$

As for the first term, we apply Assumption N2(i), (18) and Lemma 3. Then

$$
\mathcal{J}_{1}=\frac{k_{n} q_{n}}{n} \sigma_{n}^{2}(\mathbf{x}, t) \rightarrow 0 \text { as } n \rightarrow+\infty
$$

Regarding the second term, by stationarity we can write

$$
\begin{aligned}
\mathcal{J}_{2} & =\frac{2 k_{n}}{n} \sum_{l=1}^{q_{n}-1}\left(q_{n}-l\right)\left|\operatorname{cov}\left(\tilde{\Psi}_{1}(\mathbf{x}, t), \tilde{\Psi}_{l+1}(\mathbf{x}, t)\right)\right| \\
& \leq \frac{2 k_{n} q_{n}}{n} \sum_{l=1}^{q_{n}-1}\left|\operatorname{cov}\left(\tilde{\Psi}_{1}(\mathbf{x}, t), \tilde{\Psi}_{l+1}(\mathbf{x}, t)\right)\right|
\end{aligned}
$$




$$
\begin{aligned}
& \leq \frac{2 k_{n} q_{n}}{n} q_{n} \frac{1}{h_{1}^{d} l^{2}(\mathbf{x})}\left|\operatorname{cov}\left(\Psi_{1}(\mathbf{x}, t), \Psi_{l+1}(\mathbf{x}, t)\right)\right| \\
& \leq m_{1} \frac{k_{n} q_{n}}{n} q_{n} h_{1}^{d} .
\end{aligned}
$$

From the second part of Lemma 3 and assumptions N2(i)-(ii), we get that $\mathcal{J}_{2} \rightarrow 0$ as $n \rightarrow+\infty$. (ii) Using stationarity and Theorem 5.3, p.89 in [BULINSKI and SHASHKIN 2007] we have

$$
\begin{aligned}
& \frac{1}{n}\left|\operatorname{cov}\left(U_{n 1}^{\prime}, U_{n, l+1}^{\prime}\right)\right|=\frac{1}{n}\left|\sum_{i=p_{n}+1}^{p_{n}+q_{n}} \sum_{i=l\left(p_{n}+q_{n}\right)+p_{n}+1}^{(l+1)\left(p_{n}+q_{n}\right)} \operatorname{cov}\left(\tilde{\Psi}_{i}(\mathbf{x}, t), \tilde{\Psi}_{j}(\mathbf{x}, t)\right)\right| \\
& =\frac{1}{n}\left|\sum_{r=1}^{q_{n}} \sum_{j=l\left(p_{n}+q_{n}\right)+p_{n}+1}^{(l+1)\left(p_{n}+q_{n}\right)} \operatorname{cov}\left(\tilde{\Psi}_{p_{n}+r}(\mathbf{x}, t), \tilde{\Psi}_{j}(\mathbf{x}, t)\right)\right| \\
& =\frac{1}{n} \mid \sum_{r=1}^{q_{n}}\left(q_{n}-r+1\right) \operatorname{cov}\left(\tilde{\Psi}_{1}(\mathbf{x}, t), \tilde{\Psi}_{l\left(p_{n}+q_{n}\right)+r}(\mathbf{x}, t)\right) \\
& +\sum_{r=1}^{q_{n}-1}\left(q_{n}-r\right) \operatorname{cov}\left(\tilde{\Psi}_{r+1}(\mathbf{x}, t), \tilde{\Psi}_{l\left(p_{n}+q_{n}\right)+1}(\mathbf{x}, t)\right) \mid \\
& =\frac{1}{n} \mid \sum_{r=1}^{q_{n}}\left(q_{n}-r+1\right) \operatorname{cov}\left(\tilde{\Psi}_{1}(\mathbf{x}, t), \tilde{\Psi}_{l\left(p_{n}+q_{n}\right)+r}(\mathbf{x}, t)\right) \\
& +\sum_{r=1}^{q_{n}-1}\left(q_{n}-r\right) \operatorname{cov}\left(\tilde{\Psi}_{1}(\mathbf{x}, t), \tilde{\Psi}_{l\left(p_{n}+q_{n}\right)-r+1}(\mathbf{x}, t)\right) \mid \\
& \leq \frac{q_{n}}{n} \sum_{r=l\left(p_{n}+q_{n}\right)+1}^{l\left(p_{n}+q_{n}\right)+q_{n}}\left|\operatorname{cov}\left(\tilde{\Psi}_{1}(\mathbf{x}, t), \tilde{\Psi}_{r}(\mathbf{x}, t)\right)\right| \\
& +\frac{q_{n}}{n} \sum_{r=l\left(p_{n}+q_{n}\right)-\left(q_{n}-2\right)}^{l\left(p_{n}+q_{n}\right)}\left|\operatorname{cov}\left(\tilde{\Psi}_{1}(\mathbf{x}, t), \tilde{\Psi}_{r}(\mathbf{x}, t)\right)\right| \\
& =\frac{q_{n}}{n} \sum_{r=l\left(p_{n}+q_{n}\right)-\left(q_{n}-2\right)}^{l\left(p_{n}+q_{n}\right)+q_{n}}\left|\operatorname{cov}\left(\tilde{\Psi}_{1}(\mathbf{x}, t), \tilde{\Psi}_{r}(\mathbf{x}, t)\right)\right| \\
& =\frac{q_{n}}{n} \sum_{r=l\left(p_{n}+q_{n}\right)-\left(q_{n}-1\right)}^{l\left(p_{n}+q_{n}\right)+\left(q_{n}-1\right)}\left|\operatorname{cov}\left(\tilde{\Psi}_{1}(\mathbf{x}, t), \tilde{\Psi}_{r+1}(\mathbf{x}, t)\right)\right| \\
& =\frac{q_{n}}{n h_{1}^{d} l^{2}(\mathbf{x})} \sum_{r=l\left(p_{n}+q_{n}\right)-\left(q_{n}-1\right)}^{l\left(p_{n}+q_{n}\right)+\left(q_{n}-1\right)}\left|\operatorname{cov}\left(\Psi_{1}(\mathbf{x}, t), \Psi_{r+1}(\mathbf{x}, t)\right)\right| \\
& =\frac{q_{n} M_{0}}{n h_{1}^{d+2} h_{2}^{2} l^{2}(\mathbf{x})} \sum_{r=l\left(p_{n}+q_{n}\right)-\left(q_{n}-1\right)}^{l\left(p_{n}+q_{n}\right)+\left(q_{n}-1\right)}\left|\Lambda_{1, r+1}(\mathbf{x}, t)\right|
\end{aligned}
$$


where $M_{0}=\max \left\{h_{2} \operatorname{Lip}(K)\|K\|_{\infty}^{d-1}, h_{1}\left(\operatorname{Lip}(H)+h_{2} \frac{\operatorname{Lip}(\bar{G})}{\bar{G}(\tau)}\right)\left\|K_{d}\right\|_{\infty}\right\}$ and (ii) is then obtained.

(iii) By stationarity and (ii), we find

$$
\begin{aligned}
\frac{1}{n} \sum_{1 \leq i<j \leq k_{n}}\left|\operatorname{cov}\left(U_{n i}^{\prime}, U_{n j}^{\prime}\right)\right| & =\frac{1}{n} \sum_{l=1}^{k_{n}-1}\left(k_{n}-l\right)\left|\operatorname{cov}\left(U_{n 1}^{\prime}, U_{n, l+1}^{\prime}\right)\right| \\
& \leq \frac{k_{n}}{n} \sum_{l=1}^{k_{n}-1}\left|\operatorname{cov}\left(U_{n 1}^{\prime}, U_{n, l+1}^{\prime}\right)\right| \\
& \leq m_{1} \frac{q_{n} k_{n}}{n} \frac{1}{l^{2}(\mathbf{x}) h_{1}^{d+2} h_{2}^{2}} \sum_{l=1}^{k_{n}-1} \sum_{r=l\left(p_{n}+q_{n}\right)-\left(q_{n}-1\right)}\left|\Lambda_{1, r+1}(\mathbf{x}, t)\right| \\
& \leq m_{\frac{q_{n}}{k_{n}}} \frac{1}{n} \frac{1}{l^{2}(\mathbf{x}) h_{1}^{d+2} h_{2}^{2}} \sum_{r=p_{n}}^{\infty}\left|\Lambda_{1, r+1}(\mathbf{x}, t)\right| \\
& \leq m_{1} \frac{q_{n} k_{n}}{n} \frac{1}{l^{2}(\mathbf{x})} \frac{\gamma_{0}}{1-e^{-\gamma}} \frac{e^{-\gamma p_{n}}}{h_{1}^{d+2} h_{2}^{2}} \rightarrow 0 .
\end{aligned}
$$

The result follows from assumptions N2(i) and (iii).

LEMMA 5- If Assumption N2 holds, we have

(i) $\frac{k_{n}}{n} \operatorname{var}\left(U_{n 1}\right) \rightarrow \sigma^{2}(\boldsymbol{x}, t)$

(ii) $\frac{1}{n}\left|\operatorname{cov}\left(U_{n 1}, U_{n, l+1}\right)\right| \leq \frac{p_{n} m_{1}}{n l^{2}(\boldsymbol{x})} \frac{1}{h_{1}^{d+2} h_{2}^{2}} \sum_{r=l\left(p_{n}+q_{n}\right)-p_{n}}^{l\left(p_{n}+q_{n}\right)+p_{n}}\left|\Lambda_{1, r+1}(\boldsymbol{x}, t)\right|$

(iii) $\frac{1}{n} \sum_{1 \leq i<j \leq k_{n}}\left|\operatorname{cov}\left(U_{n i}, U_{n j}\right)\right| \rightarrow 0$

(iv) $\operatorname{var}\left(\frac{T_{n}}{\sqrt{n}}\right) \rightarrow \sigma^{2}(\boldsymbol{x}, t)$

\subsection{Proof of Lemma 5}

(i) We have

$$
\begin{aligned}
\frac{k_{n}}{n} \operatorname{var}\left(U_{n 1}\right) & =\frac{k_{n}}{n} \operatorname{var}\left(\sum_{i=1}^{p_{n}} \tilde{\Psi}_{i}(\mathbf{x}, t)\right) \\
& =\frac{p_{n} k_{n}}{n} \frac{1}{h_{1}^{d} l^{2}(\mathbf{x})} \operatorname{var}\left(\Psi_{1}(\mathbf{x}, t)\right)+\frac{2 k_{n}}{n h_{1}^{d} l^{2}(\mathbf{x})} \sum_{1 \leq i<j \leq p_{n}}\left|\operatorname{cov}\left(\Psi_{i}(\mathbf{x}, t), \Psi_{j}(\mathbf{x}, t)\right)\right|
\end{aligned}
$$

By the same arguments as in Lemma 4(i) and Assumption N2(i)-(ii), we get the result.

(ii) An analogous framework as in Lemma 4(ii) gives

$$
\frac{1}{n}\left|\operatorname{cov}\left(U_{n 1}, U_{n, l+1}\right)\right|=\frac{1}{n}\left|\sum_{i=1}^{p_{n}} \sum_{i=l\left(p_{n}+q_{n}\right)+1}^{l\left(p_{n}+q_{n}\right)+p_{n}} \operatorname{cov}\left(\tilde{\Psi}_{i}(\mathbf{x}, t), \tilde{\Psi}_{j}(\mathbf{x}, t)\right)\right|
$$




$$
\begin{aligned}
= & \frac{1}{n} \mid \sum_{r=1}^{p_{n}}\left(p_{n}-r+1\right) \operatorname{cov}\left(\tilde{\Psi}_{1}(\mathbf{x}, t), \tilde{\Psi}_{l\left(p_{n}+q_{n}\right)+r}(\mathbf{x}, t)\right) \\
& +\sum_{r=1}^{p_{n}-1}\left(p_{n}-r\right) \operatorname{cov}\left(\tilde{\Psi}_{r+1}(\mathbf{x}, t), \tilde{\Psi}_{l\left(p_{n}+q_{n}\right)+1}(\mathbf{x}, t)\right) \mid \\
= & \frac{1}{n} \mid \sum_{r=1}^{p_{n}}\left(p_{n}-r+1\right) \operatorname{cov}\left(\tilde{\Psi}_{1}(\mathbf{x}, t), \tilde{\Psi}_{l\left(p_{n}+q_{n}\right)+r}(\mathbf{x}, t)\right) \\
& +\sum_{r=1}^{p_{n}-1}\left(p_{n}-r\right) \operatorname{cov}\left(\tilde{\Psi}_{1}(\mathbf{x}, t), \tilde{\Psi}_{l\left(p_{n}+q_{n}\right)-r+1}(\mathbf{x}, t)\right) \mid \\
\leq & \frac{p_{n}}{n} \sum_{r=l\left(p_{n}+q_{n}\right)-\left(p_{n}-2\right)}^{l\left(p_{n}+q_{n}\right)+p_{n}} \operatorname{cov}\left(\tilde{\Psi}_{1}(\mathbf{x}, t), \tilde{\Psi}_{r}(\mathbf{x}, t)\right) \mid \\
= & \frac{p_{n}}{n h_{1}^{d} l^{2}(\mathbf{x})} \sum_{r=l\left(p_{n}+q_{n}\right)-\left(p_{n}-1\right)}^{l\left(p_{n}+q_{n}\right)+p_{n}}\left|\operatorname{cov}\left(\Psi_{1}(\mathbf{x}, t), \Psi_{r+1}(\mathbf{x}, t)\right)\right| \\
\leq & \frac{p_{n} m_{1}}{n h_{1}^{d+2} h_{2}^{2} l^{2}(\mathbf{x})} \sum_{r=l\left(p_{n}+q_{n}\right)-p_{n}}\left|\Lambda_{1, r+1}(\mathbf{x}, t)\right|
\end{aligned}
$$

(iii) By stationarity and item (ii), we have

$$
\begin{aligned}
\frac{1}{n} \sum_{1 \leq i<j \leq k_{n}}\left|\operatorname{cov}\left(U_{n i}, U_{n j}\right)\right| & =\frac{1}{n} \sum_{l=1}^{k_{n}-1}\left(k_{n}-l\right)\left|\operatorname{cov}\left(U_{n 1}, U_{n, l+1}\right)\right| \\
& \leq \frac{k_{n}}{n} \sum_{l=1}^{k_{n}-1}\left|\operatorname{cov}\left(U_{n 1}, U_{n, l+1}\right)\right| \\
& \leq m_{1} \frac{p_{n} k_{n}}{n} \frac{1}{l^{2}(\mathbf{x}) h_{1}^{d+2} h_{2}^{2}} \sum_{l=1}^{k_{n}-1} \sum_{r=l\left(p_{n}+q_{n}\right)-p_{n}}^{l\left(p_{n}+q_{n}\right)+p_{n}}\left|\Lambda_{1, r+1}(\mathbf{x}, t)\right| \\
& \leq m \frac{p_{n} k_{n}}{n} \frac{1}{l^{2}(\mathbf{x}) h_{1}^{d+2} h_{2}^{2}} \sum_{r=p_{n}}^{\infty}\left|\Lambda_{1, r+1}(\mathbf{x}, t)\right| \\
& \leq m_{1} \frac{p_{n} k_{n}}{n} \frac{1}{l^{2}(\mathbf{x})} \frac{\gamma_{0}}{1-e^{-\gamma}} \frac{e^{-\gamma q_{n}}}{h_{1}^{d+2} h_{2}^{2}} \rightarrow 0 .
\end{aligned}
$$

As stated in Lemma 4(ii) and from Assumptions N2(i) and (iii), we get the result. (iv) Observe that

$$
\begin{aligned}
\operatorname{var}\left(\frac{T_{n}}{\sqrt{n}}\right) & =\frac{1}{n} \operatorname{var}\left(\sum_{m=1}^{k_{n}} U_{n m}\right) \\
& =\frac{k_{n}}{n} \operatorname{var}\left(U_{n 1}\right)+\frac{2}{n} \sum_{1 \leq i<j \leq k_{n}}\left|\operatorname{cov}\left(U_{n i}, U_{n j}\right)\right|
\end{aligned}
$$


By items (i) and (iii), we finish the proof of Lemma 5.

As for the demonstration of (23), we use exactly the same arguments as those employed in Lemma 5(iii)

$$
\begin{aligned}
\operatorname{var}\left(\frac{T_{n}^{\prime}}{\sqrt{n}}\right) & =\frac{1}{n} \operatorname{var}\left(\sum_{m=1}^{k_{n}} U_{n m}^{\prime}\right) \\
& =\frac{k_{n}}{n} \operatorname{var}\left(U_{n 1}^{\prime}\right)+\frac{2}{n} \sum_{1 \leq i<j \leq k_{n}}\left|\operatorname{cov}\left(U_{n i}^{\prime}, U_{n j}^{\prime}\right)\right| \rightarrow 0 .
\end{aligned}
$$

This holds by items (i) and (iii) of Lemma 4. And,

$$
\begin{aligned}
\operatorname{var}\left(\frac{T_{n}{ }^{\prime \prime}}{\sqrt{n}}\right) & =\frac{1}{n} \operatorname{var}\left(U_{n k_{n}}^{\prime \prime}\right) \\
& =\frac{1}{n} \operatorname{var}\left(\sum_{l=k_{n}\left(p_{n}+q_{n}\right)+1}^{n} \tilde{\Psi}_{l}(\mathbf{x}, t)\right) \\
& \left.=\frac{n-k_{n}\left(p_{n}+q_{n}\right)}{n} \operatorname{var}\left(\tilde{\Psi}_{1}(\mathbf{x}, t)\right)+\frac{2}{n} \sum_{k_{n}\left(p_{n}+q_{n}\right)+1 \leq i<j \leq n} \mid \operatorname{cov}\left(\tilde{\Psi}_{i}(\mathbf{x}, t)\right), \tilde{\Psi}_{j}(\mathbf{x}, t)\right) \mid \\
& =: \mathcal{I}_{n 1}+\mathcal{I}_{n 2} .
\end{aligned}
$$

We may write

$$
\begin{aligned}
\mathcal{I}_{n 1} & =\frac{n-k_{n}\left(p_{n}+q_{n}\right)}{n} \frac{1}{h_{1}^{d} l^{2}(\mathbf{x})} \operatorname{var}\left(\Psi_{1}(\mathbf{x}, t)\right) \\
& \leq \frac{p_{n}}{n} \sigma_{n}^{2}(\mathbf{x}, t) .
\end{aligned}
$$

Employing Assumption N2 and Lemma 3, we obtain that $\mathcal{I}_{n 1}=o(1)$. Remark that $n-k_{n}\left(p_{n}+q_{n}\right) \leq p_{n}$. As for the second term, we have

$$
\begin{aligned}
\mathcal{I}_{n 2} & \left.=\frac{2}{n} \sum_{k_{n}\left(p_{n}+q_{n}\right)+1 \leq i<j \leq n} \mid \operatorname{cov}\left(\tilde{\Psi}_{i}(\mathbf{x}, t)\right), \tilde{\Psi}_{j}(\mathbf{x}, t)\right) \mid \\
& \left.=\frac{2}{n} \sum_{1 \leq i<j \leq n-k_{n}\left(p_{n}+q_{n}\right)} \mid \operatorname{cov}\left(\tilde{\Psi}_{i}(\mathbf{x}, t)\right), \tilde{\Psi}_{j}(\mathbf{x}, t)\right) \mid \quad \text { (by stationarity) } \\
& \left.\leq \frac{2}{n} \sum_{1 \leq i<j \leq p_{n}} \mid \operatorname{cov}\left(\tilde{\Psi}_{i}(\mathbf{x}, t)\right), \tilde{\Psi}_{j}(\mathbf{x}, t)\right) \mid \\
& \left.=\frac{2}{n} \sum_{l=1}^{p_{n}-1}\left(p_{n}-l\right) \mid \operatorname{cov}\left(\tilde{\Psi}_{1}(\mathbf{x}, t)\right), \tilde{\Psi}_{l+1}(\mathbf{x}, t)\right) \mid \\
& \left.\leq \frac{2 p_{n}}{n} \sum_{l=1}^{p_{n}-1} \mid \operatorname{cov}\left(\tilde{\Psi}_{1}(\mathbf{x}, t)\right), \tilde{\Psi}_{l+1}(\mathbf{x}, t)\right) \mid \\
& \leq \frac{p_{n}\left(p_{n}-1\right)}{n h_{1}^{d} l^{2}(\mathbf{x})} m_{1} h_{1}^{2 d}
\end{aligned}
$$




$$
=O\left(\frac{p_{n}}{n} p_{n} h_{1}^{d}\right)
$$

Thus, under Assumption N2 the proof of (23) is achieved.

The next step consists in proving (22), that is by proving first that the rv's $\left\{U_{n m}, m=1, \ldots, k\right\}$ are asymptotically independent. For this purpose, we deal with the characteristic functions and show that

$$
\left|\mathbb{E}\left(e^{i t \sum_{m=1}^{k_{n}} \frac{1}{\sqrt{n}} U_{n m}}\right)-\prod_{m=1}^{k_{n}} \mathbb{E}\left(e^{i t \frac{1}{\sqrt{n}} U_{n m}}\right)\right| \rightarrow 0 \text { as } n \rightarrow \infty
$$

Indeed, we have

$$
\begin{aligned}
& I_{k_{n}}(t)=:\left|\mathbb{E}\left(e^{i t \sum_{m=1}^{k_{n}} \frac{U_{n m}}{\sqrt{n}}}\right)-\prod_{m=1}^{k_{n}} \mathbb{E}\left(e^{i t \frac{U_{n m}}{\sqrt{n}}}\right)\right| \\
& =\left|\mathbb{E}\left(e^{i t \sum_{m=1}^{k_{n}} \frac{U_{n m}}{\sqrt{n}}}\right)-\prod_{m=1}^{k_{n}-1} \mathbb{E}\left(e^{i t \frac{U_{n m}}{\sqrt{n}}}\right)\left(e^{i t \frac{1}{\sqrt{n}} U_{n k_{n}}}\right)\right| \\
& \leq\left|\mathbb{E}\left(e^{i t \sum_{m=1}^{k_{n}} \frac{U_{n m}}{\sqrt{n}}}\right)-\mathbb{E}\left(e^{i t \frac{U_{n k_{n}}}{\sqrt{n}}}\right) \mathbb{E}\left(e^{i t \sum_{m=1}^{k_{n}-1} \frac{U_{n m}}{\sqrt{n}}}\right)\right| \\
& +\left|\mathbb{E}\left(e^{i t \frac{U_{n k_{n}}}{\sqrt{n}}}\right) \mathbb{E}\left(e^{i t \sum_{m=1}^{k_{n}-1} \frac{U_{n m}}{\sqrt{n}}}\right)-\prod_{m=1}^{k_{n}-1} \mathbb{E}\left(e^{i t \frac{U_{n m}}{\sqrt{n}}}\right) \mathbb{E}\left(e^{i t \frac{U_{n k_{n}}}{\sqrt{n}}}\right)\right| \\
& =\left|\mathbb{E}\left(e^{i t \frac{U_{n k_{n}}}{\sqrt{n}}}\right)\right|\left|\mathbb{E}\left(e^{i t \sum_{m=1}^{k_{n}-1} \frac{U_{n m}}{\sqrt{n}}}\right)-\prod_{m=1}^{k_{n}-1} \mathbb{E}\left(e^{i t \frac{U_{n m}}{\sqrt{n}}}\right)\right| \\
& +\left|\mathbb{E}\left(e^{i t \sum_{m=1}^{k_{n}} \frac{U_{n m}}{\sqrt{n}}}\right)-\mathbb{E}\left(e^{i t \frac{U_{n k_{n}}}{\sqrt{n}}}\right) \mathbb{E}\left(e^{i t \sum_{m=1}^{k_{n}-1} \frac{U_{n m}}{\sqrt{n}}}\right)\right| \\
& =\left|\mathbb{E}\left(e^{i t \sum_{m=1}^{k_{n}-1} \frac{U_{n m}}{\sqrt{n}}}\right)-\prod_{m=1}^{k_{n}-1} \mathbb{E}\left(e^{i t \frac{U_{n m}}{\sqrt{n}}}\right)\right| \\
& +\left|\mathbb{E}\left(e^{i t \sum_{m=1}^{k_{n}} \frac{U_{n m}}{\sqrt{n}}}\right)-\mathbb{E}\left(e^{i t \frac{U_{n k_{n}}}{\sqrt{n}}}\right) \mathbb{E}\left(e^{i t \sum_{m=1}^{k_{n}-1} \frac{U_{n m}}{\sqrt{n}}}\right)\right| \\
& =: I_{k_{n}-1}(t)+\left|\operatorname{cov}\left(e^{i t \sum_{m=1}^{k_{n}-1} \frac{U_{n m}}{\sqrt{n}}}, e^{i t \frac{U_{n k_{n}}}{\sqrt{n}}}\right)\right| \text {. }
\end{aligned}
$$

Analogously $I_{k_{n}-1}(t) \leq I_{k_{n}-2}(t)+\left|\operatorname{cov}\left(e^{i t \sum_{m=1}^{k_{n}-2} \frac{U_{n m}}{\sqrt{n}}}, e^{i t \frac{U_{n k_{n}-1}}{\sqrt{n}}}\right)\right|$

Therefore

$$
\begin{aligned}
\left|\mathbb{E}\left(e^{i t \sum_{m=1}^{k_{n}} \frac{U_{n m}}{\sqrt{n}}}\right)-\prod_{m=1}^{k_{n}} \mathbb{E}\left(e^{i t \frac{U_{n m}}{\sqrt{n}}}\right)\right| \leq & \left|\operatorname{cov}\left(e^{i t \sum_{m=1}^{k_{n}-1} \frac{U_{n m}}{\sqrt{n}}}, e^{i t \frac{U_{n k_{n}}}{\sqrt{n}}}\right)\right| \\
& +\left|\operatorname{cov}\left(e^{i t \sum_{m=1}^{k_{n}-2} \frac{U_{n m}}{\sqrt{n}}}, e^{i t \frac{U_{n k_{n}-1}}{\sqrt{n}}}\right)\right| \\
& +\ldots+\left|\operatorname{cov}\left(e^{i t \frac{U_{n 1}}{\sqrt{n}}}, e^{i t \frac{U_{n 2}}{\sqrt{n}}}\right)\right| .
\end{aligned}
$$


In what follows, we apply the Lemma1 in [BULINSKI 1996] on each term in the right hand side of (24). For this purpose, we need to calculate the first order partial derivatives of the function $\mathcal{V}_{m}: \mathbb{R}^{p_{n}(d+1)} \rightarrow$ $\mathbb{R}, m=1, \ldots, k_{n}$ defined by $V_{m}\left(\mathbf{x}_{l}, y_{l}\right)=e^{i t \frac{U_{n m}}{\sqrt{n}}}$ for all $l$ lying between $(m-1)\left(p_{n}+q_{n}\right)+1$ and $(m-1)\left(p_{n}+q_{n}\right)+p_{n}$, where

$$
\mathbf{x}_{l}=\left(x_{(m-1)\left(p_{n}+q_{n}\right)+1}^{1}, \ldots, x_{(m-1)\left(p_{n}+q_{n}\right)+1}^{d}, \ldots, x_{(m-1)\left(p_{n}+q_{n}\right)+p_{n}}^{1}, \ldots, x_{(m-1)\left(p_{n}+q_{n}\right)+p_{n}}^{d}\right)
$$

and

$$
y_{l}=\left(y_{(m-1)}\left(p_{n}+q_{n}\right)+1, \ldots, y_{(m-1)}\left(p_{n}+q_{n}\right)+p_{n}\right) .
$$

By the way, the first partial derivative of the function $\mathcal{V}_{m}$ with respect to $y_{l}$ for $l=(m-1)\left(p_{n}+q_{n}\right)+$ $1, \ldots,(m-1)\left(p_{n}+q_{n}\right)+p_{n}$ is given by

$$
\begin{aligned}
\frac{\partial \mathcal{V}_{m}}{\partial y_{l}}\left(\mathbf{x}_{l}, y_{l}\right)= & \frac{\partial}{\partial y_{l}}\left(i t \frac{U_{n m}}{\sqrt{n}}\right) e^{i t \frac{U_{n m}}{\sqrt{n}}} \\
= & \frac{\partial}{\partial y_{l}}\left(\frac{i t}{\sqrt{n}} \sum_{i=(m-1)\left(p_{n}+q_{n}\right)+1}^{(m-1)\left(p_{n}+q_{n}\right)+p_{n}} \tilde{\Psi}_{l}\right) e^{i t \frac{U_{n m}}{\sqrt{n}}} \\
= & \frac{i t}{\sqrt{n}} e^{i t \frac{U_{n m}}{\sqrt{n}}} \frac{\partial}{\partial y_{l}}\left[\frac{1}{\sqrt{h_{1}^{d}} l(\mathbf{x})}\left(K_{d, l}\left[H_{l} \frac{\delta_{l}}{\bar{G}\left(Y_{l}\right)}-F(t \mid \mathbf{x})\right]\right)\right] \\
= & \frac{i t}{\sqrt{n h_{1}^{d}} l(\mathbf{x})} e^{i t \frac{U_{n m}}{\sqrt{n}}} K_{d}\left(\frac{\mathbf{x}-\mathbf{x}_{l}}{h_{1}}\right) \delta_{l} \frac{\partial}{\partial y_{l}}\left[H\left(\frac{t-y_{l}}{h_{2}}\right) \frac{1}{\bar{G}\left(y_{l}\right)}\right] \\
= & \frac{i t}{\sqrt{n h_{1}^{d} h_{2}^{2}} l(\mathbf{x})} e^{i t \frac{U_{n m}}{\sqrt{n}}} K_{d}\left(\frac{\mathbf{x}-\mathbf{x}_{l}}{h_{1}}\right) \\
& \times \delta_{l}\left[-h_{2} \frac{g\left(y_{l}\right)}{\bar{G}^{2}\left(y_{l}\right)} H\left(\frac{t-y_{l}}{h_{2}}\right)-H^{(1)}\left(\frac{t-y_{l}}{h_{2}}\right) \frac{1}{\bar{G}\left(y_{l}\right)}\right]
\end{aligned}
$$

Moreover, the first partial derivative of the function $\mathcal{V}_{m}$ with respect to $x_{l}^{j}$ for $l=(m-1)\left(p_{n}+q_{n}\right)+$ $1, \ldots,(m-1)\left(p_{n}+q_{n}\right)+p_{n}$ and $j=1, \ldots d$ is

$$
\begin{aligned}
\frac{\partial \mathcal{V}_{m}}{\partial x_{l}^{j}}\left(\mathbf{x}_{l}, y_{l}\right)= & \frac{i t}{\sqrt{n h_{1}^{d}}} e^{i t \frac{U_{n m}}{\sqrt{n}}}\left[\frac{H_{l} \delta_{l}}{\bar{G}\left(y_{l}\right)}-F(t \mid \mathbf{x})\right] \frac{\partial}{\partial x_{l}^{j}}\left[\frac{1}{l(\mathbf{x})} K_{d}\left(\frac{\mathbf{x}-\mathbf{x}_{l}}{h_{1}}\right)\right] \\
= & \frac{i t}{\sqrt{n h_{1}^{d}}} e^{i t \frac{U_{n m}}{\sqrt{n}}}\left[\frac{H_{l} \delta_{l}}{\bar{G}\left(y_{l}\right)}-F(t \mid \mathbf{x})\right] \\
& \times\left[-\frac{l^{(1)}(\mathbf{x})}{l^{2}(\mathbf{x})} K_{d}\left(\frac{\mathbf{x}-\mathbf{x}_{l}}{h_{1}}\right)+\frac{\partial}{\partial x_{l}^{j}} K_{d}\left(\frac{\mathbf{x}-\mathbf{x}_{l}}{h_{1}}\right) \frac{1}{l(\mathbf{x})}\right]
\end{aligned}
$$

Hence, we get

$$
\begin{aligned}
\frac{\partial \mathcal{V}_{m}}{\partial x_{l}^{j}}\left(\mathbf{x}_{l}, y_{l}\right)= & \frac{i t}{\sqrt{n h_{1}^{d+2}}} e^{i t \frac{U_{n m}}{\sqrt{n}}}\left[\frac{H_{l} \delta_{l}}{\bar{G}\left(y_{l}\right)}-F(t \mid \mathbf{x})\right] \\
& \times\left[-h_{1} \frac{l^{(1)}(\mathbf{x})}{l^{2}(\mathbf{x})} K_{d}\left(\frac{\mathbf{x}-\mathbf{x}_{l}}{h_{1}}\right)-\prod_{i=1, i \neq j}^{d} K\left(\frac{x^{i}-x_{l}^{i}}{h_{1}}\right) K^{(1)}\left(\frac{x^{j}-x_{l}^{j}}{h_{1}}\right)\right]
\end{aligned}
$$


Besides, Applying assumptions A2-A4 and A7, there exists $A>0$ such that

$$
\left\|\frac{\partial \mathcal{V}_{m}}{\partial y_{l}}\left(\mathbf{x}_{l}, y_{l}\right)\right\|_{\infty} \leq \frac{A t}{\sqrt{n h_{1}^{d} h_{2}^{2}}} \text { and }\left\|\frac{\partial \mathcal{V}_{m}}{\partial x_{l}^{j}}\left(\mathbf{x}_{l}, y_{l}\right)\right\|_{\infty} \leq \frac{A t}{\sqrt{n h_{1}^{d+2}}}
$$

Thereby, using the Lemma 1 in [BULINSKI 1996] we find

$$
\left|\operatorname{cov}\left(e^{i t \frac{U_{n 2}}{\sqrt{n}}}, e^{i t \frac{U_{n 1}}{\sqrt{n}}}\right)\right| \leq \frac{A^{2} t^{2}}{n h_{1}^{d+1} h_{2}} \sum_{i \in I_{1}} \sum_{j \in I_{2}}\left|\Lambda_{i, j}(\mathbf{x}, t)\right| .
$$

In addition

$$
\left|\operatorname{cov}\left(e^{i t \sum_{m=1}^{k_{n}-1} \frac{U_{n m}}{\sqrt{n}}}, e^{i t \frac{U_{n k_{n}}}{\sqrt{n}}}\right)\right| \leq \frac{A^{2} t^{2}}{n h_{1}^{d+1} h_{2}} \sum_{i \in I_{1}+\ldots+I_{k_{n}-1}} \sum_{j \in I_{k_{n}}}\left|\Lambda_{i, j}(\mathbf{x}, t)\right| .
$$

We conclude

$$
\begin{aligned}
\left|\mathbb{E}\left(e^{i t \sum_{m=1}^{k_{n}} \frac{U_{n m}}{\sqrt{n}}}\right)-\prod_{m=1}^{k_{n}} \mathbb{E}\left(e^{i t \frac{U_{n m}}{\sqrt{n}}}\right)\right| \leq & \frac{A^{2} t^{2}}{n h_{1}^{d+1} h_{2}}\left[\sum_{i \in I_{1}} \sum_{j \in I_{2}}\left|\Lambda_{i, j}(\mathbf{x}, t)\right|+\sum_{i \in I_{1}+I_{2}} \sum_{j \in I_{3}}\left|\Lambda_{i, j}(\mathbf{x}, t)\right|\right. \\
& \left.+\ldots+\sum_{i \in I_{1}+\ldots+I_{k_{n}-1}} \sum_{j \in I_{k_{n}}}\left|\Lambda_{i, j}(\mathbf{x}, t)\right|\right]
\end{aligned}
$$

Using stationarity, the inequality (25) becomes

$$
\begin{aligned}
\left|\mathbb{E}\left(e^{i t \sum_{m=1}^{k_{n}} \frac{U_{n m}}{\sqrt{n}}}\right)-\prod_{m=1}^{k_{n}} \mathbb{E}\left(e^{i t \frac{U_{n m}}{\sqrt{n}}}\right)\right| \leq & \frac{A^{2} t^{2}}{n h_{1}^{d+1} h_{2}}\left[\left(k_{n}-1\right) \sum_{i \in I_{1}} \sum_{j \in I_{2}}\left|\Lambda_{i, j}(\mathbf{x}, t)\right|\right. \\
& \left.+\ldots+\sum_{i \in I_{1}} \sum_{j \in I_{k_{n}}}\left|\Lambda_{i, j}(\mathbf{x}, t)\right|\right]
\end{aligned}
$$

Again, by stationarity we have

$$
\begin{aligned}
\left|\mathbb{E}\left(e^{i t \sum_{m=1}^{k_{n}} \frac{U_{n m}}{\sqrt{n}}}\right)-\prod_{m=1}^{k_{n}} \mathbb{E}\left(e^{i t \frac{U_{n m}}{\sqrt{n}}}\right)\right| & \leq \frac{A^{2} t^{2}}{n h_{1}^{d+1} h_{2}} p_{n} k_{n} \sum_{j=\left(k_{n}-1\right)\left(p_{n}+q_{n}\right)-\left(p_{n}-2\right)}^{\left(k_{n}-1\right)\left(p_{n}+q_{n}\right)+p_{n}}\left|\Lambda_{1, j}(\mathbf{x}, t)\right| \\
& \leq m_{1} t^{2} \frac{p_{n} k_{n}}{n} \frac{1}{h_{1}^{d+1} h_{2}} \sum_{j=q_{n}}^{\infty}\left|\Lambda_{1, j}(\mathbf{x}, t)\right| \\
& \leq m_{1} t^{2} \frac{p_{n} k_{n}}{n} \frac{\gamma_{0}}{1-e^{-\gamma}} \frac{e^{-\gamma q_{n}}}{h_{1}^{d+1} h_{2}} \rightarrow 0 .
\end{aligned}
$$

Note that (26) tends to zero under assumptions N2(i) and (iii).

It remains to show that $\frac{T_{n}}{\sqrt{n}}$ is asymptotically normal, we rely on the standard Lindeberg's condition

$$
k_{n} \mathbb{E}\left(\frac{1}{n} U_{n 1}^{2} \mathbb{1}_{\left\{\left|\frac{1}{\sqrt{n}} U_{n 1}\right|>\varepsilon \sigma(\mathbf{x}, t)\right\}}\right) \rightarrow 0 .
$$


From assumption A2, we can write

$$
\begin{aligned}
\left|U_{n 1}\right| & =\left|\sum_{m=1}^{p_{n}} \frac{1}{\sqrt{h_{1}^{d}} l(\mathbf{x})}\left\{K_{d, i}\left[H_{i} \frac{\delta_{i}}{\bar{G}\left(Y_{i}\right)}-F(t \mid \mathbf{x})\right]-\mathbb{E}\left[K_{d, i}\left(H_{i} \frac{\delta_{i}}{\bar{G}\left(Y_{i}\right)}-F(t \mid \mathbf{x})\right)\right]\right\}\right| \\
& \leq \frac{2 p_{n}\left\|K_{d}\right\|_{\infty}}{\sqrt{h_{1}^{d}} l(\mathbf{x}) \bar{G}\left(\tau_{F}\right)} .
\end{aligned}
$$

Therefore, by Tchebytchev's inequality and assumptions A4 and N2(ii) we get

$$
\begin{aligned}
k_{n} \mathbb{E}\left(\frac{1}{n} U_{n 1}^{2} \mathbb{1}_{\left.\left\{\left|\frac{1}{\sqrt{n}} U_{n 1}\right|>\varepsilon \sigma(\mathbf{x}, t)\right\}\right)}\right. & \leq \frac{4 k_{n} p_{n}^{2}\left\|K_{d}\right\|_{\infty}^{2}}{n h_{1}^{d} l^{2}(\mathbf{x}) \bar{G}^{2}\left(\tau_{F}\right)} \mathbb{P}\left(\frac{1}{\sqrt{n}}\left|U_{n 1}\right|>\varepsilon \sigma(\mathbf{x}, t)\right) \\
& \leq \frac{4\left\|K_{d}\right\|_{\infty}^{2}}{l^{2}(\mathbf{x}) \bar{G}^{2}\left(\tau_{F}\right)} \frac{k_{n} \operatorname{Var}\left(U_{n 1}\right)}{n \varepsilon^{2} \sigma^{2}(\mathbf{x}, t)} \frac{p_{n}^{2}}{n h_{1}^{d}} \rightarrow 0 .
\end{aligned}
$$

This achieves the proof of Theorem 1.

\subsection{Proof of Corollary 1}

Making use of a Taylor expansion of $F_{n}(. \mid$.$) in the neighborhood of \xi_{p}$, we get

$$
\xi_{p, n}(\mathbf{x})-\xi_{p}(\mathbf{x})=\frac{F_{n}\left(\xi_{p, n}(\mathbf{x}) \mid \mathbf{x}\right)-F_{n}\left(\xi_{p}(\mathbf{x}) \mid \mathbf{x}\right)}{f_{n}\left(\xi_{p, n}^{*}(\mathbf{x}) \mid \mathbf{x}\right)}
$$

where $\xi_{p, n}^{*}$ lies between $\xi_{p}$ and $\xi_{p, n}$. The almost sure convergence of $\xi_{p, n}(\mathbf{x})$ to $\xi_{p}(\mathbf{x})$ [see DJELLADJ and TATACHAK 2019], Proposition 1 and the continuity of $f(. \mid$.) give the convergence in probability of $f_{n}\left(\xi_{p, n}^{*}(\mathbf{x}) \mid \mathbf{x}\right)$ toward $f\left(\xi_{p}(\mathbf{x}) \mid \mathbf{x}\right)$. The proof is hence established using Theorem 1 .

\section{Bibliographie}

BULINSKI A., On the convergence rates in the central limit theorem for positively and negatively dependent random fields. In : bragimov, I. A. and Zaitsev, A. Yu. (eds.), Probab. Theory and Math. Statist. (1996) : 3-14.

BUlinski A., SHAShKin A., Limit theorems for associated random fields and related systems. Singapore World Scientific, 2007.

DJelladj W., TAtachak A., Strong uniform consistency of a kernel conditional quantile estimator for censored and associated data. Hacettepe Journal of Mathematics and Statistics 48 (2019) : 290-311.

Doukhan P., Neumann M., Probability and moment inequalities for sums of weakly dependent random variables, with applications. Stochastic Processes and their Applications 117 (2007) : 878-903.

Esary J., Proschan F., Walkup D., Association of random variables with applications. Ann. Math. Stat. 38 (1967) : 1466-1476.

FerRAni Y., OUld SAID E., TATACHAK A., On kernel density and mode estimates for associated and censored data. Communications in Statistics - Theory and Methods, 45 (2016) : 1853-1862.

Kaplan E.L., Meier P., Nonparametric estimation from incomplete observations. J. Am. Statist. Assoc. 53 (1958) : 457-481. 
Menni N., Tatachak A., A note on estimating the conditional expectation under censoring and association : strong uniform consistency. Stat. Papers 59 (2018):1009-1030.

OULD SAID E., A strong uniform convergence rate of kernel conditional quantile estimator under random censorship. Statist. Probab. Letters 76 (2006) : 579-586.

OULD SAID E., SADKi O., Asymptotic normality for a smooth kernel estimator of the conditional quantile for censored time series. South African Statistical Journal 45 (2011) :65-98.

Ould SAId E., SAdKi O., Prediction via the conditional quantile for right censorship model. Far East Journal of Theoretical Statistics 25 (2008) :145-179.

Yu K., Jones M.C., Local linear quantile regression. J. Am. Stat. Assoc. 93 (1998) : 228-238. 